\title{
Targeted senolytic prodrug is well tolerated and results in amelioration of frailty, muscle regeneration and cognitive functions in geriatric mice
}

\section{Linda Doan}

Rubedo Life Sciences

Patrick Paine

Lausanne University

Christopher Tran

Stanford University

Benjamin Parsons

Rubedo Life Sciences

Amanda Hiller

Rubedo Life Sciences

Isaac Joshua

Rubedo Life Sciences

Nicholas Collaco

Rubedo Life Sciences

Alberto Vitari

Rubedo Life Sciences

Antoine De Morree

Stanford University

Heather Ishak

Stanford University

Jeffrey Jasper

Rubedo Life Sciences

Mark Gallop

Rubedo Life Sciences

Jian Luo

Stanford University

\section{Tony Wyss-Coray}

Stanford University https://orcid.org/0000-0001-5893-0831

Thomas Rando

Stanford University 
Marco Quarta ( $\square$ marcoquarta@rubedolife.com )

Rubedo Life Sciences

Article

Keywords: targeted senolytic prodrug, senescent cells, geriatric mice, age-related disease

Posted Date: October 20th, 2020

DOI: https://doi.org/10.21203/rs.3.rs-92962/v1

License: (c) (1) This work is licensed under a Creative Commons Attribution 4.0 International License. Read Full License 
Manuscript Title

\section{Targeted senolytic prodrug is well tolerated and results in amelioration of frailty, muscle regeneration and cognitive functions in geriatric mice}

Linda Doan ${ }^{1,2}$, Patrick Paine ${ }^{1,2,3}$, Christopher Tran², Benjamin Parsons ${ }^{1}$, Amanda Hiller ${ }^{1}$, Isaac Joshua ${ }^{1}$, Nicholas Collaco ${ }^{1}$, Alberto C. Vitari ${ }^{1}$, Antoine De Morree ${ }^{2,4}$, Heather Ishak ${ }^{2}$, Jeffrey R. Jasper ${ }^{1}$, Mark A. Gallop ${ }^{1}$, Jian Luo ${ }^{2,4,5}$, Tony Wyss-Coray ${ }^{2,4,5}$, Thomas A. Rando $2,4,5$, Marco Quarta $1,2,4,5,6$ *

${ }^{1}$ Rubedo Life Sciences, Sunnyvale, California, USA

${ }^{2}$ Department of Neurology and Neurological Sciences, Stanford University School of Medicine, Stanford, California, USA.

${ }^{3}$ Department of Pharmacology and Toxicology, Lausanne University, $\mathrm{CH}$.

${ }^{4}$ Paul F. Glenn Center for the Biology of Aging, Stanford University School of Medicine, Stanford, California, USA.

${ }^{5}$ Center for Tissue Regeneration, Repair and Restoration, Veterans Affairs Palo Alto Health Care System, Palo Alto, California, USA.

${ }^{6}$ Molecular Medicine Research Institute, Sunnyvale, California USA

*Correspondence to marcoquarta@rubedolife.com 


\begin{abstract}
Mammalian aging coincides with an accumulation of senescent cells. Depletion of senescent cells shows promise to treat age-related diseases. However, many of the senolytic drugs used to deplete senescent cells cause profound toxic side effects. Here we tested a prodrug form of a pan-cytotoxic drug and demonstrated dramatically reduced side effects in geriatric mice while gaining senolytic selectivity. Specifically, we employ a strategy that takes advantage of the greatly enriched expression of the lysosomal hydrolase $\beta$-Galactosidase in senescent cells as a tool to selectively convert the prodrug into the active parent drug. In young, old and geriatric mice this prodrug was well tolerated and proved capable of reducing the burden of senescence in multiple tissues without evident toxicity. Importantly, chronic systemic treatment of geriatric mice resulted in reduced frailty index, improved muscle tissue and stem cell functions, improved cognitive functions and overall improved survival. This work supports future senolytic prodrug designs, based on other metabolic functions characteristic of senescent cells, on different parent drugs and aimed at clinical development. This new class of senolytic prodrugs promises capabilities of targeting selectively and safely specific senescent cell types in multiple tissues to treat age-related diseases in geriatric people.
\end{abstract}




\section{Introduction}

Frailty is one of the most relevant clinical syndromes in geriatric patients ${ }^{1,2}$, which entangles older adults in a vicious cycle of energy dysregulation ${ }^{3}$ leading to loss of weight, strength and balance, exhaustion, low exercise capacity, and slow, unstable walking. The outcome is an increased state of vulnerability, which diminishes the ability of the body to respond to stress, thus reducing physiological reserves and impairing the ability of the body to maintain homeostasis ${ }^{4}$. In frail individuals, an apparently small insult (e.g., introduction of a new medication; minor infection; or minor surgery) can result in a dramatic and disproportionate change in health state: from independent to dependent; mobile to immobile; postural stability to falling; lucid to delirious ${ }^{5-7}$. Frailty is also linked with cognitive decline, dementia and Alzheimer's disease $(A D)^{4,5}$. Although Frailty has been recognized by the World Health Organization ${ }^{1}$ and the U.S. National Academy of Sciences $^{8}$ as a major health concern among the elderly, an effective therapeutic solution is still not available.

Frailty is connected to cellular aging, which in turn is connected to cellular senescence $^{9}$. Senescent cells are permanently withdrawn from the cell cycle and generally develop a persistent pro-inflammatory phenotype called the senescence-associated secretory phenotype (SASP) which is comprised of proinflammatory cytokines and chemokines ${ }^{10}$. Accumulation of senescent cells has been observed at sites of many agerelated injuries ${ }^{11}$. Studies confirmed that injecting senescent cells into young mice increased frailty and mortality ${ }^{12}$. Inducing apoptosis of senescent cells in vivo delays deterioration of the vasculature, heart, and kidney, and it replenishes the hematopoietic stem cell pool restoring tissue homeostasis after chemotoxicity and aging ${ }^{13-16}$. The efficacy of this treatment strongly depends on the subtype of the senescent cells targeted, being most pronounced against senescent cells with a high inflammatory status ${ }^{17}$. 
Selective killing of senescent cells with therapeutics (i.e., senolytics) have gained attention as a new therapeutic approach for age-related diseases. Targeting of prosurvival Senescent Cell Anti-apoptotic Pathways (SCAPs) ${ }^{18}$ has emerged as the primary strategy for senescent cell killing. Until now, the reported classes of senolytics include kinase inhibitors, $\mathrm{Bcl}-2$ family inhibitors, natural products, a p53 binding inhibitor, heat shock protein 90 (HSP90) inhibitors, UBX0101, a histone deacetylase (HDAC) inhibitor, cardiac glycosides and bromodomain and extra-terminal domain (BET) family protein inhibitors ${ }^{19}$. The combined administration of dasatinib and quercetin (targeting BCL-2/BCL$\mathrm{XL}, \mathrm{PI} 3 \mathrm{~K} / \mathrm{AKT}$, p53/p21/serpine and the dependence receptors/tyrosine kinase SCAP) by clearing senescent cells in the tissues has been shown to improve the pathologies of diverse age-related diseases, including cardiac aging ${ }^{19}$, atherosclerosis ${ }^{20}$, osteoporosis ${ }^{21}$, pulmonary fibrosis ${ }^{22}$, hepatic steatosis ${ }^{23}$, and Alzheimer's disease ${ }^{24}$. However, the general lack of specificity towards senescent cells ${ }^{25,26}$ often leads to hematological toxicities including neutropenia ${ }^{27}$ and thrombocytopenia ${ }^{28}$. Moreover, mTOR inhibitors have found their way into senolytic application since an inhibitory action on SCAPS has been described $^{29}$. Again, their translational value in vivo is limited due to their chronic toxicity, which leads to hyperglycemia, hyperlipidemia, insulin resistance and increased incidence of new-onset type 2 diabetes ${ }^{30}$. These specificity issues of current senolytics limits their application to local delivery and/or to acute treatments as opposed to chronic systemic administration. The identification of agents that selectively kill senescent cells while sparing other cell populations represents a scientific challenge. Current senolytic drugs target molecular pathways shared between senescent and proliferating cells, thus achieving cell killing but not specificity. As a matter of fact, many known senolytic agents were initially developed as cytotoxic anti-cancer agents and subsequently repurposed for 'selective' removal of senescent cell populations. Because proliferating cells are frequently more sensitive to the cytotoxic or cytostatic effect of anti-tumor agents, dose-limiting 
toxicity, especially in rapidly replicating hematopoietic, skin or gut cells, is a frequently observed side-effect, which strongly limits the clinical utility of these anti-senescence therapies ${ }^{27}$. Pulsatile administration of senolytic drugs has been proposed as a mechanism to minimize exposure of non-senescent cells and therefore off-target effects ${ }^{31}$. However, this approach likely limits therapeutic efficacy due to restricted dosing regimens, particularly in elderly individuals affected by geriatric syndrome. Typically, this patient population is frail, affected by comorbidities and do not tolerate the side effects sustained by younger individuals. Currently, there remains an unmet need to identify senolytics that can be targeted to senescent cells safely, selectively and systemically.

Senescent cells are characterized by a notable change in biological properties such as an increase in the levels of mitochondria, reactive oxygen species, lysosomal content, and upregulation of many lysosomal proteins, including the lysosomal enzyme senescence-associated $\beta$-galactosidase $(S A-\beta G a l)^{32,33}$. Increased activity of SA- $\beta$ gal is detected not only in cells undergoing senescence in vitro, but also in the tissues from aged animals and humans ${ }^{34-36}$. Recently, a promising strategy has been proposed based on galactose-derivative prodrugs. These prodrugs are selectively activated in senescent cells upon conversion into the parent active drug by the hydrolase activity of SA- $\beta$ Gal. In particular, specific senotoxic compounds such as duocarmycin, gemcitabine and navitoclax have been modified into galacto-derivative prodrugs showing increased selectivity in targeting senescence cells and efficacy in treating cancer and aged mouse models ${ }^{37-39}$

Here, we report a novel prodrug design to target senescent cells, allowing systemic removal of senescent cells in geriatric mice without noticeable side effects. We took advantage of the senescence-specific activity of SA- $\beta$ Gal in the design of a non-toxic senolytic prodrug derivative of the compound 5-Fluorouridine, a metabolic precursor of the clinically approved anti-cancer medication 5-Fluorouracil. We first tested the specificity of 
this prodrug on senescent cells in vitro. We then confirmed safety and efficacy of the prodrug in young (5 month-old), aged (22 month old) and in geriatric (30 month old) mice. Importantly, we showed that geriatric mice that received the prodrug treatment for four weeks altogether improved significantly: 1) frailty profile; 2) skeletal muscle function; 3) muscle stem cell function; 4) cognitive function; and 5) survival. Prodrug senolytic pharmacological treatments are a promising strategy to overcome the current limitations in chronic and systemic treatments of older individuals affected with neurodegenerative diseases, age-related diseases and geriatric syndromes.

\section{Results}

\section{Generation of 5FURGal Prodrug.}

Design of the senolytic prodrug employed in this study begins with a small molecule having intrinsic senotoxicity, and then chemically conjugates at an appropriate site within the molecule the hexose sugar galactose to generate a $\beta$-D-galactopyranosyl adduct such that the cytotoxicity of the parent molecule is masked. The inactive prodrug is therefore transformed into its cytotoxic parent only within senescent cells after hydrolytic cleavage of the $\beta$-D-galactosyl promoiety by the abundantly expressed SA- $\beta$ Gal enzyme (FIG 1a). A small library of cytotoxic chemotherapeutic compounds amenable for chemical modification as described was assembled. In vitro screening was performed to verify the ability of these small molecules to induce apoptosis in human senescent fibroblasts. 5Fluorouridine $(5 \mathrm{FU})$ is a well-characterized chemotherapeutic agent, which exerts its cytotoxic action on cancer cells by interfering with DNA replication through inhibition of thymidylate synthase. Interestingly, $5 \mathrm{FU}$ also showed the ability to induce apoptosis in senescent cells by interfering with RNA synthesis ${ }^{40}$. This compound is known for its high level of toxicity in vivo and was chosen to test the capacity of the prodrug derivation to prevent its side effects while retaining senolytic efficacy. Following our prodrug design 
concept, we modified 5FU into the inactive prodrug 5-fluorouridine-5'-O- $\beta-D-$

galactopyranoside (5FURGal) and proceeded to validate its senolytic activity and safety in vitro and in vivo (Fig. 1b).

\section{FURGal prodrug selectively kills senescent cells in vitro.}

We first tested the specificity of the 5FURGal prodrug. In particular, we evaluated whether the pro-apoptotic effects of the parent drug 5FU against proliferating or quiescent human fibroblasts were blunted for prodrug 5FURGal, while maintaining senotoxic efficacy of the latter against senescent fibroblast cells.

As anticipated, while the cytotoxic potency of $5 \mathrm{FU}$ was greatest against proliferating human IMR90 fibroblasts $\left(\mathrm{IC}_{50} \sim 0.0015 \mathrm{mM}\right)$, substantial toxicity of the parent drug was also observed against quiescent or senescent fibroblast cells $\left(\mathrm{IC}_{50}=0.07\right.$ and $0.039 \mathrm{mM}$ respectively). By contrast, we found that 5FURGal showed minimal toxicity against either proliferating fibroblasts $\left(\mathrm{IC}_{50} \sim 4.4 \mathrm{mM}\right)$ or quiescent fibroblasts $\left(\mathrm{IC}_{50}>10 \mathrm{mM}\right)$ but retained pro-apoptotic activity toward senescent IMR90 cells $\left(\mathrm{IC}_{50} \sim 0.30 \mathrm{mM}\right.$ ) as intended (Fig 1ce). To confirm the hypothesized mechanism of action that 5FURGal is specifically hydrolyzed by SA- $\beta$ Gal, we employed human fibroblasts where the gene GLB1 encoding $\beta$ Gal has been knocked out. As predicted, we found that $\beta-G a l$ deficient senescent cells were significantly less sensitive to $5 \mathrm{FURGal}\left(\mathrm{IC}_{50}>10 \mathrm{mM}\right)$ than $\beta$-Gal proficient senescent cells $\left(\mathrm{IC}_{50} \sim 0.30 \mathrm{mM}\right)$, suggesting loss of their ability to convert $5 \mathrm{FURGal}$ into parent drug $5 \mathrm{FU}$ (Fig. f-g). Finally, to explore non-specific effects of 5FURGal on the cell cycle, we cultured proliferative or senescent fibroblasts, with or without 5FURGal and in the presence of EdU, a thymidine analog that is incorporated during the S Phase of the cell cycle. We found that 5FURGal had no impact on cell cycle phases in either cell population (Supp. Fig. 1). Taken together, these results show that $5 F U R G a l$ is efficiently converted into $5 F U$ in senescent cells but not in proliferating or quiescent cells, suggesting that prodrug 5FURGal might be a 
suitable candidate senolytic molecule with high selectivity for senescent cells and minimal toxicity for non-senescent cells.

\section{FURGal is well tolerated in vivo.}

To evaluate whether the selectivity of 5FURGal can prevent the toxicity of the parent drug 5FU, we tested the effects of both compounds in vivo in four month-old C57/b6 mice. We found that 5FURGal did not replicate the severe acute toxicity of 5FU observed after IP administration. In particular, dosing mice with $(100 \mathrm{mg} / \mathrm{kg}) 5 \mathrm{FU}$ resulted in: a) loss of body weight (Fig. 1h); b) loss of spleen mass (Fig. 1i), loss of bone marrow cell count (Fig. 1l), and c) thrombocytopenia, neutropenia, and lymphocytopenia (Figure $1 \mathrm{~m}$ ). Conversely, mice treated with an equimolar dose of 5FURGal $(160 \mathrm{mg} / \mathrm{Kg})$, similar to vehicle-treated control mice group, showed no deleterious impact on any of the parameters analyzed, suggesting that 5FURGal is not acutely toxic in the tissues tested. Moreover, pharmacokinetic studies demonstrate that mice dosed intravenously with 5FURGal show low levels of systemic conversion of prodrug to the parent drug 5FU ( $2 \%$ on a dose-adjusted basis) (Supp Fig. 2). Taken together, these results indicate that 5FURGal could be safely administered systemically with minimal toxicity and high tolerability in young mice.

\section{A luciferin prodrug is employed to assess senescence-associated bioluminescence in aged mouse models.}

To test the senolytic efficacy of 5FURGal, we investigated its ability to target senescent cells in vivo. First, we developed a novel preclinical murine model to assess senescence levels non-invasively using a prodrug strategy similar to 5FURGal. Young (4 months) and aged (22 months) transgenic mice constitutively expressing the bioluminescent enzyme Luciferase (FVB-Tg(CAG-luc,-GFP)L2G85Chco/J or FVB-LucGFP) were used to develop a method of senescence-associated bioluminescence imaging (SA-BLI). To 
achieve this, we employed LUCGal, a $\beta$-D-galactosyl conjugated prodrug version of luciferin (the luciferase substrate). Similar to 5FURGal, LUCGal is hydrolyzed selectively in cells where expression and activity of SA- $\beta$ Gal are highly enriched (Fig. 2a). Bioimaging of these mice showed that aged mice have $\sim 6$ fold higher levels of bioluminescence compared to young mice, suggesting increased levels of $S A-\beta G a l^{+}$cells associated with age (Fig. $2 b$ and c). As a positive control, we treated a cohort of young mice with the chemotherapeutic agent Doxorubicin ('chemo mouse' model), reported to induce cellular senescence in vivo and capable of promoting an accelerated aging phenotype in cancer patients ${ }^{41,42}$. We found that the levels of SA-BLI in chemo mice were similar to naturally aged mice (Fig. 2b and c). Next, from the same mice previously employed for the SA-BLI studies we isolated liver tissue to confirm levels of senescence histologically. We performed colorimetric staining for SA- $\beta$ Gal in sections of liver and we found that, similar to the SA-BLI results, young mice exhibited only baseline levels of SA- $\beta$ Gal. Conversely, both chemo mice and naturally aged mice showed $\sim 800$-fold higher levels of SA- $\beta$ Gal compared to baseline, indicating accumulation of senescent cells (Fig. 2d and e). These results were consistent with gene expression levels of Cdkn2A ( $\mathrm{p} 16^{\mathrm{INK} 4 \mathrm{~A}}$ ), another reported marker of cellular senescence. We performed qPCR in liver samples isolated from the same mice, and we found that $\mathrm{p} 16^{\text {INK4A }}$ was upregulated 16 fold in naturally aged mice and young chemo mice compared to untreated young mice (Fig. 2f). Taken together, these results suggest that a prodrug derivative of luciferin can be effectively employed to achieve selective SA-BLI in aged mice as a preclinical model to study cellular senescence.

\section{FURGal shows senolytic and functional efficacy in aged mice.}

To test efficacy of the prodrug 5FURGal for senolytic and functional activity in vivo, we evaluated the overall physical performance for aged FVB-LucGFP mice and correlated 
this with SA-BLI levels. To this end, we measured grip strength, endurance, balance and spatial learning memory and combined the results into a performance score. We found that this performance score inversely correlates with SA-BLI levels in aged FVB-LucGFP mice, suggesting that in aged mice higher levels of senescence are associated with lower physiological performance (Fig. 3a). Next, we treated aged FVB-LucGFP mice via intraperitoneal (IP) injections with two doses (separated by two days) for two weeks of: a) 5FURGal at $140 \mathrm{mg} / \mathrm{Kg}$; or b) saline. Mice treated with 5FURGal showed a reduction of SABLI when measured 3 days following the last injection (Figure $1 \mathrm{~b}$ and $\mathrm{c}$ ). Next, the day after SA-BLI was measured we tested the effect of 5FURGal treatment on physiological functions. Compared to aged mice treated with saline, aged mice treated with 5FURGal showed improved grip strength, running time and learning spatial cognitive functions (Fig. $3 \mathrm{~d}$-f). To confirm prior SA-BLI results, we histologically assessed SA- $\beta$ Gal and we found lower levels in livers of aged mice treated with 5FURGal (Fig. 3h, i). Similarly, p16 ${ }^{\text {INK4A }}$ gene expression levels were reduced in kidney and liver tissues (Figure $1 \mathrm{~g}$ and I), further suggesting that 5FURGal treatment results in clearance of senescent cells in vivo. To confirm these results in a different model of in vivo senescence, we employed the chemo mouse model. Chemo mice that received a treatment with 5FURGal 4 and 6 days after the last Doxorubicin injection presented reduced levels of SA- $\beta$ Gal staining in the liver compared to chemo mice treated with saline (Supp. Fig. 3a,b). Taken together, these results suggest that 5FURGal is capable of reducing senescent cell levels in vivo in multiple tissues and following different senescence induction stimuli. Finally, to test the beneficial senolytic effects of 5FURGal in a model different from natural aged mice, chemo mice were employed as a model of accelerated aging. When treated with saline, chemo mice showed significant weight loss 8 days following Doxorubicin dosing. However, 5FURGal treatment increased the tolerability for Doxorubin treatment with a significant attenuation of weight loss (Supp. Fig. 2c). Taken together, these results suggest that the beneficial senolytic effects of 5FURGal in aged mice 
models lead to recovery of youthful healthy physiological traits.

\section{FURGal reverts physiological traits of Frailty in geriatric mice.}

Next, we aimed to test whether 5FURGal was able to reduce the burden of Frailty in geriatric mice. With this goal in mind, we chose a model of aged-related Frailty Syndrome which includes loss of certain skeletal muscle functions. In particular, we employed a cohort of geriatric (30 month-old) Pax $7^{\mathrm{CreER}} / \mathrm{flox}$-YFP mice, that express the yellow fluorescent protein (YFP) specifically in muscle stem cells upon induction with Tamoxifen. We employed this cohort of geriatric mice for extensive longitudinal studies on physiological and cognitive functions. To comprehensively study the effects of 5FURGal on geriatric mice, the experiment was designed in three phases: 1) Frailty Assessment; 2) Cognitive Assessment; 3) Skeletal Muscle Function Assessment (Fig. 4a). The mice were randomly divided into two groups, one to be treated with saline (vehicle control) and one with 5FURGal (dose 140 $\mathrm{mg} / \mathrm{Kg}$ ), twice per week for four weeks in total, with a week of rest in between (Fig. 4a). In Phase One, we assessed the mice functionally before and after the treatment. A characteristic of Frailty is exhaustion and overall weakness, which results in decreased mobility that is considered to be one of the best predictors of age-related loss of functions ${ }^{43}$. To test this, mice were housed individually in cages with access to computer-controlled running wheels during the entire duration of Phase One. We found that geriatric mice treated with 5FURGal significantly improved their spontaneous activity, quantified as total distance run over time. Conversely, geriatric mice treated with saline showed the opposite trend (Fig. 4b and Supp Fig. 4a). These results suggest that 5FURGal treatment improves activity levels in geriatric mice.

To generate a Frailty index, we measured 10 different parameters in young mice to set the youthful baseline level (defined as $100 \%$ score, where each parameter was weighted 
for $10 \%$ of the score). In particular, we measured: 1) body weight; 2) motor coordination; 3 ) endurance; 4) dystonia; 5) ataxia; 6) grip strength; 7) spontaneous activity; 8) cognitive functions; 9) gait; and 10) coat condition. Frailty score was assessed in the geriatric mice cohort before treatment, and we found that they scored an average $\sim 30 \%$ (geriatric level) compared to the youthful basal level. However, when we repeated the measurements following treatments, we found that geriatric mice receiving 5FURGal improved their Frailty Score, averaging $\sim 60 \%$, while saline-treated geriatric mice did not show improved scores (Fig. 4c). Moreover, despite the small cohort analyzed, geriatric mice treated with 5FURGal showed a trend of $\sim 40 \%$ higher survival and lower mortality compared to mice treated with saline (Fig. 4d). Taken together these results suggest that systemic senolytic treatment with prodrug 5FURGal results in amelioration of the Frailty profile and decreased mortality in geriatric mice.

\section{Prodrug treatment improves cognitive functions in geriatric mice.}

Loss of memory and loss of spatial orientation are two aspects characteristic of agerelated dementia and Alzheimer's disease (AD) which are often comorbid with Frailty Syndrome ${ }^{44,45}$. In Phase Two, we investigated 5FURGal properties in reversing cognitive decline. To measure spatial learning, we employed the $Y$ Maze test. Mice were tested before and after treatment. Treated mice with 5FURGal improved their spontaneous alternation performance significantly compared to mice treated with saline, suggesting improved spatial learning functions (Fig. 5a and Supp Fig. 5a-b). We then performed the Morris Water Maze test to measure learning memory and memory retrieval. Geriatric mice treated with 5FURGal showed shorter latencies to escape onto the hidden platform and spend more time in the target quadrant in the probe test performing more similarly to young mice, suggesting improved memory functions (Fig. 5b, c). The loss of cognitive functions associated with age 
is linked to morphological changes in the hippocampus, where microglial cells' activation increases ${ }^{46,47}$. Brains of mice were fixed, sectioned and immune-stained with antibodies against IBA-1 (marker of microglia cells) and CD68 (marker of activated microglia). We found that increased levels of microglia and activated microglia observed in the hippocampal brain regions of geriatric mice treated with saline were significantly reduced after 5FURGal treatment and more similar to brains of young mice (Fig.5 d-f). Taken together, these results show that systemic senolytic treatment with 5FURGal improved cognitive functions and reduced neuroinflammation in geriatric mice.

\section{Prodrug treatment restores skeletal muscle regenerative capacity and stem cell functions in geriatric mice.}

Frailty Syndrome is associated with loss of muscle strength and sarcopenia, which is associated with loss of muscle stem cell (MuSC) function ${ }^{48,49}$. In Phase Three, we measured skeletal muscle functions, including MuSC functions and muscle regenerative capacity after inducing an injury performing intramuscular injections of Cardiotoxin (CTX) into tibialis anterior (TA) muscles. First, to trace MuSCs over time, we injected young and geriatric mice with Tamoxifen to induce the recombination and expression of the YFP protein driven by the Pax7CreEr locus, which is selectively expressed by MuSCs. Then, to assess muscle contractile properties, we performed ex vivo electrophysiological biomechanics measurements of dissected TA muscles 10 days after CTX injury. We found that TA muscles from geriatric mice treated with saline generated $\sim 60 \%$ less contractile tetanic force compared to muscles from young mice treated with saline. Conversely, geriatric mice treated with 5FURGal significantly improved tetanic force generation compared to saline treatments (Fig. 6a). We then asked if the improved functionality of skeletal muscle was associated with improved regenerative capacity. To that end, we performed immunohistochemistry on 
sections of the left TA muscles analyzed previously for contractile properties. Morphometry analysis showed that the cross-sectional area of $\mathrm{YFP}^{+}$centrally nucleated myofibers, a hallmark of regenerated myofibers, was significantly increased in geriatric mice treated with 5FURGal compared to geriatric mice treated with saline (Fig. 6b, c).

Next, we wanted to explore whether the improved muscle regenerative capacity was associated with improved MuSCs functions. Following muscle function assessment, YFP ${ }^{+}$ MuSCs were isolated from the right TA muscles by fluorescence-activated cell sorting (FACS). With age, MuSCs decline in number and in their proliferative potential ${ }^{50}$. We found that MuSCs isolated from geriatric mice treated with 5FURGal were higher in number compared to treatment with saline (Fig. 6d). Moreover, when these MuSCs were cultured in vitro, they exhibited enhanced proliferative capacity analyzed in a single cell myoclonal formation assay and by quantification of EdU ${ }^{+}$YFP ${ }^{+}$MuSCs, after culturing them with EDU

(Fig. 6e-f). Taken together, these results indicate that systemic senolytic treatment of 5FURGal in geriatric mice improves skeletal muscle functions, MuSC functions and regenerative capacity.

\section{Chronic systemic treatment with prodrug is well tolerated in geriatric mice.}

Finally, we determined the tolerability profile of 5FURGal dosed chronically in geriatric mice. We thus performed toxicology studies in a separate cohort of geriatric mice (C57/b6 mice 28-30 months old). Geriatric mice received a total of five tail vein injections with $130 \mathrm{mg} / \mathrm{Kg}$ of 5 FURGal or saline over a three-week period. Similar to the Pax7CreEr/YFP mice cohort, no significant difference in body weight was detected in the two groups (Supp. Fig. 6a). Additionally, 5FURGal treatment did not negatively affect mice survival over the course of the three-week treatment (Supp. Fig. 6b). Spleen was dissected and analyzed at the end of the experiment (day 21). Although no statistical difference was 
measured between 5FURGal or saline treatments, the former showed a trend toward smaller spleen sizes, similar to young mice. Conversely, geriatric mice treated with saline showed aged-related enlarged spleen sizes (Supp. Fig. 6b). The hematology profile of blood isolated from both groups at day 21 did not show significant differences across a multitude of hematopoietic cell types (Supp. Fig. 6c). Taken together, these results suggest that, regardless of the elevated toxicity of the parent drug $5 \mathrm{FU}$, the prodrug $5 F U R G a l$ is well tolerated in geriatric mice and can be safely administered systemically and chronically without evident side effects.

\section{Discussion}

Frail old individuals often present with one or more comorbidities, including myocardial infarction, peripheral vascular disease, stroke, cancer, diabetes mellitus, arthritis, and chronic lung disease ${ }^{51}$. Due to their altered health status they cannot tolerate the side effects previously described for current senolytic therapies. For this reason, most of the current senolytic drugs are under development for single aged-related conditions. We explored a prodrug-based approach to increase the selectivity and the tolerability of a senolytic agent, with the goal of developing strategies for safe systemic therapeutics. In particular, we took advantage of the well-known SA- $\beta-$ Gal, an hydrolase enzyme enriched in the lysosomal compartment of senescent cells and employed as a marker for cellular senescence. To this end, we employed a 5'-O- $\beta$-D-galactopyranosyl modification of the toxic nucleoside 5-fluorouridine 5FUR to generate the selective senolytic prodrug 5FURGal, and have investigated this molecule in various rodent models of age-related pathologies. We found that the prodrug 5FURGal was able to kill human senescent cells but spared proliferative or quiescent cells in vitro. When tested in vivo, young, old and geriatric mice tolerated the prodrug well with no evident toxic effects. Conversely, the parent drug 5FU, a 
toxic chemotherapeutic agent, is poorly tolerated in young mice and not tolerated in aged mice. As a senolytic, prodrug 5FURGal reduced the level of senescence analyzed through multiple methods, including a non-invasive in vivo bioluminescent assessment based on the same galacto-derivative prodrug strategy. Finally, efficacy studies of 5FURGal in geriatric mice demonstrated that the prodrug reduces the frailty profile while improving: 1) muscle tissue and stem cell functions; 2) cognitive functions; 3) and overall mouse survival. Taken together, our results show that using a sugar derivative prodrug-based strategy we can turn a pan-cytotoxic drug into a safe and effective senolytic. This strategy supports a new generation of senolytic drugs to overcome current limitations in treating elderly individuals with geriatric syndromes.

Aging is often characterized by skeletal muscle catabolism leading to sarcopenia and frailty. Sarcopenia is a syndrome characterized by progressive loss of skeletal muscle mass and function related to aging, disease, starvation or disuse, and typically precedes frailty. Frailty is an age-related syndrome comprising states such as exhaustion, weakness, and slowness, and more broadly, cognition. Recent studies indicate a significant relationship between age-associated declines in both physical and cognitive function ${ }^{52}$. Importantly, frailty can be considered to be a state of diminished resolution of homeostasis following a stressor event that increases the risk of adverse health outcomes, including fall-related injuries and even delirium.

Accumulation of senescent cells in aging and the resultant release of SASPassociated factors (pro-inflammatory cytokines, chemokines, growth factors, immune modulators and proteases) have been linked with various diseases of aging ${ }^{53}$. Increases in IL-54,55, MCP-1 ${ }^{56,57}$, and p16INK4a ${ }^{58,59}$ are associated with frailty in humans. Notably, in preclinical studies of aging and function, transplantation of small numbers of senescent cells (autologous ear fibroblasts, non-autologous preadipocytes or human preadipocytes) into the 
abdominal cavity was sufficient to induce physical dysfunction in young mice ${ }^{12}$. Senescent cells were also detected in skeletal muscle, suggesting that senescence can spread to distant tissues, possibly induced by the SASP factors. The impairments in maximal walking speed, hanging endurance, and grip strength that occurred following the senescent cell transplantations lasted for months. Using the combination of the senolytics, dasatinib plus quercetin $(D+Q)$, senescent cell burden was decreased in the mice, and treatment of senescent cell-transplanted mice with even a single 5 day-course of $D+Q$ improved physical function in the mice for several months. When $D+Q$ was administered to aged mice (20 months old) the expression of SASP factors was decreased and physical function was improved (increased muscle strength, walking speed and physical endurance, daily activity and food intake). The combination of the senolytics $D+Q$ has further been shown to decrease the number of naturally-occurring senescent human adipose cells in tissue explants and their secretion of pro-inflammatory cytokines related to frailty ${ }^{12}$.

Building on preclinical data supporting senolytic therapy for frailty-related parameters, senolytic therapy has advanced to an ongoing clinical evaluation in healthy postmenopausal women $^{60}$. Oral dosing of the natural product Fisetin, a weakly potent, yet safe senolytic flavonoid compound, is being evaluated for the ability to help reduce markers of inflammation, insulin resistance, bone resorption and physical dysfunction in elderly women displaying gait disturbances. The primary outcome measures include: a) Improved sixminute walk time; and b) Improved gait speed. Positive findings in this pilot study could set the stage for larger clinical studies exploring the effects of Fisetin and other future senolytic drugs on age-related dysfunction.

Despite promising advancements with the early clinical trials to test effects of senolytic drugs in elderly individuals, potent and safe drugs to be administered chronically in geriatric patients are still an unmet need. Existing drugs that have been previously FDA 
approved for cancer-related clinical indications and employed in treating younger patient populations have been proposed for senolytic treatments. However, despite their proven senolytic potency in vitro for certain cell types, these drugs pose significant risks for older individuals, especially in the case of systemic exposure. In particular, small molecules such as kinase inhibitors or Bcl-2 family inhibitors present limitations including side effects that are not well tolerated in the elderly. Moreover, the lack of sufficient selectivity for cellular senescence poses additional risks of safety and efficacy. To overcome these limitations, new classes of small molecules based on prodrug strategies have been proposed as senolytics. In particular, the use of galactose-derivatized warheads has been suggested as a viable strategy to increase selectivity of small molecules toward senescent cells. Different prodrugs have been recently suggested based on this strategy: 1) a galactose-modified version of duocarmycin, a DNA-alkylating agent, has been proposed to increase the senolytic selectivity $\left.{ }^{37} ; 2\right)$ galacto-conjugation of the senolytic agent ABT-263 also called Navitoclax, a Bcl-2 class inhibitor developed for cancer therapy, has been shown to increase the parent drug senolytic specificity and reduce the platelet toxicity ${ }^{39}$; 3) a galacto-derivative prodrug of gemcitabine, another chemotherapeutic agent, has been reported to reduce inflammation and restore physical function in 20 month old mice ${ }^{38}$. These reports are consistent with our findings that a galacto-derivative prodrug is a viable strategy to turn a pan-cytotoxic small molecule into a selective agent capable of killing senescence cells. With this goal in mind and focusing on the need of minimizing the side effects and toxicity of drugs in geriatric patients, we tested the prodrug 5FURGal, derivative of the cytotoxic agent 5FU. Our results show that this is possible. Importantly, not only young or older mice but also geriatric mice tolerated 5FURGal without noticeable side effects. That allowed us to treat geriatric mice, typically frail and very sensitive to compound side effects, dosing them with chronic systemic regimens that resulted in improved physiological functions at multiple levels, including cognitive and muscular functions, and overall improved survival. 


\section{Conclusions}

We believe that observations and strategies developed during this study will serve as a valuable precursor to generating novel and more potent prodrugs for systemic senolytic applications in the clinic. The galactose-derivative prodrug 5FURGal evaluated in this study as a tool compound has an improved safety profile due to its superior specificity against senescent cells. However, beyond SA- $\beta-$ Gal, we have identified other metabolic functions that are preferentially enriched in senescent cells. In our drug development endeavours, we are currently employing these activities to produce a new generation of prodrugs designed for clinical use. Importantly, these novel prodrugs are based on parent drugs, different from $5 F U$, that we found to have intrinsic selective senolytic activity and superior potency prior to the prodrug derivation. By implementing these compounds through additional medicinal chemistry optimization we aim to eliminate specific senescent cell types accumulated during aging and pathogenic processes without affecting other cell populations, including proliferative or non-senescent quiescent cells (such as many stem cells located in their niche). This approach could allow reduction of the chronic inflammatory process associated with senescent cells, which in future clinical developments will be tested for the efficacy in reducing the burden of physical pathology (such as fibrosis or cancer), cognitive decline

(such as dementia or Alzheimer's disease) and the degeneration associated with Frailty Syndrome and other age-related diseases. 


\section{Figure Legends}

\section{Fig.1 5FURGal targets senescence without affecting proliferation and quiescence in}

vitro and toxicity in vivo. a. Schematic model of the strategy employed to generate prodrug strategy. b. Molecular structure of the prodrug 5FURGal and the parent drug 5FU. c. experimental protocol employed to test senolytic efficacy in vitro. d. IC50 values reported for human fibroblasts cell line cultured in the following conditions: 1) proliferative; 2) senescence; 3) quiescence with either 5FU or 5FURGal. e. Quantification of cell viability as indicated in panel (e) at different concentrations of either 5-FUR (left panel) or 5FURGal (right panel). f. IC50 values reported for human fibroblasts cell line KO for the hydrolase Galactosidase cultured in the following conditions: 1) proliferative; 2) senescence with either 5-FUR or 5FURGal. g. Quantification of cell viability as indicated in panel (f) at different concentrations of either 5-FUR (left panel) or 5FURGal (right panel). h-m. Tolerability study. Three separate cohorts of WT C57/B6 mice received two IP injections separated by two days of respectively: 1) saline; 2) 5FU; or 3) 5-FUR-Gal. At the terminal time point animal body weight was measured (h) before isolating and quantifying: 1) spleen weight (i); Bone marrow (I); hematopoietic cells $(m) . n=5$. Data are \pm s.e.m. For statistical analysis, t-tests were used. ${ }^{* *} P<0.0001,{ }^{* * *} P<0.00001$.

\section{Fig. 2. Prodrug based bioluminescence shows that chemotoxicity and aging are} associated with senescence burden. a. Molecular structures and enzymatic reaction steps of the prodrug Galacto-Luciferine into Oxyluciferin. b. Representative images of bioluminescence measured from mice constitutively expressing Luciferase and injected with Galacto-luciferine prodrug at: 1) 4 months of age (Young); 2) 24 months of age (Aged); or 4 months of age treated with Doxorubicin. Mice received one IP injection of Galacto-luciferine and immediately imagedc. c. Quantified results of non-invasive 
bioluminescence imaging $(n=6)$. d. Representative SA- $\beta$-Gal images from cross-sections of liver tissue after receiving treatments with saline or doxorubicin (Doxo) at different ages. e. Quantification of SA- $\beta$-Gal positive cells in liver tissue $(n=6)$. f. Quantification by qPCR of Cdkn2A gene expression from liver tissue $(n=6)$. Data are $\pm s . e . m$. For statistical analysis, t-tests were used. ${ }^{* *} P<0.0001,{ }^{* * *} P<0.00001$.

\section{Fig. 3 Prodrug senolytic treatment reduces senescence and improves physiological} functions in aged mice. a. Correlation between bioluminescence and performance. Bioluminescence in mice expressing Luciferase of 24 months of age were measured following injection of Galacto-Luciferin prodrug. Animals were then assessed for physiological functions. Specifically, measurements for grip test for limb voluntary force measurement, endurance on treadmill, balance on rotarod test, cognitive functions on $Y$ maze test were collected and combined in a performance score. Each dot represents a mouse. b. Representative images of bioluminescence measured from mice constitutively expressing Luciferase and injected with Galacto-luciferine prodrug in mice of 24 months of age (aged) treated with saline or 5-FUR-Gal prodrug. c. Quantified results of non-invasive bioluminescence imaging $(n=6)$. Functional assessment in aged mice treated with 5 FUR-Gal prodrug. Following bioluminescence imaging shown in (b and c), the same mice were assessed for: (d) voluntary limb force on grip strength test; (e) endurance on a treadmill test ; (f) spatial cognitive memory on a $Y$ maze test. Mice were assessed before and after receiving treatment with either saline or 5-FUR-Gal. g. Quantification by qPCR of Cdkn2A gene expression from kidney tissue $(n=6)$. $h$. Representative SA- $\beta-G a l$ images from cross-sections of liver tissue after receiving treatments with saline or 5-FURGal at different ages. i. Quantification of SA- $\beta-G a l$ positive cells in liver tissue $(n=6)$. I. Quantification by qPCR of Cdkn2A gene expression from liver tissue $(n=6)$. Data are \pm s.e.m. For statistical analysis, t-tests were used. ${ }^{*} \mathrm{P}<0.01,{ }^{* *} \mathrm{P}<0.001$. 
Fig. 4 Prodrug senolytic treatment reverts frailty in geriatric mice. a. Schematic of the experimental design for the frailty study. Phase 1: Young or Geriatric mice were individually housed in cages with a computer controlled running wheel and acclimated for one week before being assessed for their frailty score baseline. Geriatric mice were then divided in two cohorts. One cohort of geriatric mice and the cohort of young mice received treatments with saline. The second cohort of geriatric mice received treatment with 5FURGal. All animals received one IP injection with either saline or 5FURGal twice per week for two weeks, followed by a week of rest and another two-week of treatments. At the end of the treatments, all animals were assessed again for the frailty score. Phase 2: Following a week of rest, all mice were subjected for two weeks to Morris water maze to assess cognitive functions. Phase 3: following a week of rest, all mice received two injections of tamoxifen (TMX) separated by three days to induce recombination of the fluorescent protein YFP in MuSCs expressing Pax7. After 10 days all mice received an injection of Cardiotoxin (CTX) in both TA muscles to induce skeletal muscle injury, activation of quiescent MuSCs and the tissue regenerative process. After 10 days all animals were sacrificed, TA muscles immediately assessed for biomechanics (BM) to measure contractile properties. were then MuSCs isolated for in vitro studies or histological assessments. Black arrowheads indicate time of injections. b. Quantification of spontaneous activity measured with running wheels rotations. Total distance run is quantified for geriatric mice: 1) before initiating treatments (day 3); 2) three weeks of treatments (Day 21); 3) 5 weeks after treatments (day 35). c. Frailty Score. Young and geriatric mice were assessed for Frailty score before and after treatments with saline (young and geriatric mice) or 5FURGal (geriatric mice). Ten different parameters were measured, each weighing for up to $10 \%$ for a total of $100 \%$ based on young mice scoring (green broken line). d. Survival of geriatic mice during treatments. Geriatric mice were 
monitored during treatments with either saline or 5FURGal and their time of death recorded. Black arrowheads indicate beginning and end of treatments. Data are \pm s.e.m. For statistical analysis, t-tests were used. ${ }^{*} P<0.01,{ }^{* *} P<0.0001 . n=7$.

Fig. 5 Prodrug senolytic treatment improves cognitive functions in geriatric mice. a. Spatial Learning. Geriatric mice were subjected to $Y$ maze test before and after treatments. Graph shows fold changes of spontaneous alternation performance score $(n=$ 6). b. Learning Memory. Following treatment with saline or 5FURGal and Y Maze test, young and geriatric mice were subjected to Morris Water Maze. After one week of acclimation and training, mice swimming on a pool were assessed for their trajectory to learn the position of a platform hidden on a fixed position below the surface of the water ( $\mathrm{n}$ = 6). c. Memory Retrieval. Following the learning memory test shown in (b), mice were tested for the capacity to remember the original position of the platform removed from the pool $(n=6)$. $\mathbf{d}$. Representative images of immunofluorescence (IF) of brain cross-sections showing microglia in the hippocampal regions. Brains were isolated from the mice tested for cognitive functions assessments indicated in ( $a, b$ and $c)$, (scale bar $=2000 \mu \mathrm{m})$. IF was performed to stain nuclei (DAPI) and markers of microglia (CD68 and Iba-1). Quantification of IF for total microglia (e) and activated microglia (f) in the hippocampal regions of young or geriatric mice that received treatments with saline or 5FURGal and assessed for cognitive functions $(n=4)$. Data are \pm s.e.m. For statistical analysis, $t$-tests were used. For graph of panel (b) 2-Way ANOVA was used. ${ }^{*} P<0.01,{ }^{*} P<0.001$, ${ }^{* * *} \mathrm{P}<0.0001,{ }^{* * * *} \mathrm{P}<0.00001$. 


\section{Fig. 6 Prodrug senolytic treatment improves tissue and stem cell muscle functions}

in geriatric mice. a. Tetanic force production of young and geriatric mice treated with saline or 5FURGal. Following treatments and were tested for frailty, functional and cognitive functions, mice received an injection of CTX in the TA muscles to induce MuSCs activation and muscle regeneration. After 10 days, TA muscles were dissected and the tetanic force measured ex vivo (TA muscles from mice: young treated with saline $n=9$; geriatric treated with saline $n=5$; geriatric treated with $5 F U R G a l n=6$ ). $\mathbf{b}$. Representative IF cross-sectional images showing centrally nucleated regenerated YFP+ myofibers. Muscles from young or geriatric mice were harvested after taking force measurements (scale bar $=500 \mu \mathrm{m}) . \mathbf{c}$. Quantification of the cross-sectional area of centrally-nucleated YFP+ fibers in regenerating TA muscles that received CTS injections $(n=4)$. d. Quantification using fluorescent activated cell sorting (FACS) of MuSCs YFP+ isolated from injured TA muscles that received CTW injections (biological replicates $n=4$ ). e. Myoclonal assay of MuSCs YFP+ isolated from injured TA muscles that received CTW injections. Single MuSCs were isolated using FACS in a 96 well plate and quantified for their capacity to generate colonies (biological replicates $n=4$ ). f. Cell cycle assessment. MuSCs isolated from injured TA muscles that received CTW injections were cultured in the presence of EdU and quantified (biological replicates $n=4$ ). For statistical analysis, t-tests were used. ${ }^{*} P<0.01,{ }^{* *} P<0.001,{ }^{* * *} P<0.0001,{ }^{* * *} P<0.00001$. 


\section{Materials and Methods}

\section{Preparation of 5-Fluorouridine-5'-O- $\beta$-D-Galactopyranoside (5FURGal)}

Step 1: 5-Fluorouridine $(3.0 \mathrm{~g}, 11.4 \mathrm{mmol})$ and 2,3 dimethoxypropane $(5.1 \mathrm{~mL}, 47.8 \mathrm{mmol}$, 4.2 eq). was suspended in acetone $(9 \mathrm{~mL})$. To this was added p-toluenesulfonic acid, monohydrate (389 mg, 0.19 eq.) under a nitrogen atmosphere. After $30 \mathrm{~min}$, the mixture was a clear solution. The reaction was stirred at room temperature overnight, after which time LC/MS analysis showed complete conversion of starting material. The mixture was concentrated to dryness, ethyl acetate was added, and the solution was extracted with water $(3 \mathrm{x})$. The combined organic solvent was dried over sodium sulfate and then under high vacuum to give 5-fluoro-1-(6-(hydroxymethyl)-2,2-dimethyltetrahydrofuro[3,4d][1,3]dioxol-4-yl)pyrimidine-2,4(1H,3H)-dione as a white solid $(3 \mathrm{~g}, 86 \%$ yield) $\mathrm{m} / \mathrm{z}$ $=302.269$ found $303.5[\mathrm{M}+\mathrm{H}]^{+}$.

Step 2: 5-Fluoro-1-(6-(hydroxymethyl)-2,2-dimethyltetrahydrofuro[3,4-d][1,3]dioxol-4yl)pyrimidine-2,4(1H,3H)-dione $(3.0 \mathrm{~g}, 9.9 \mathrm{mmol})$ was dissolved in dichloromethane (160 $\mathrm{mL}$ ). To this solution was added $\mathrm{Ag}_{2} \mathrm{O}(4.35 \mathrm{~g}, 19.3 \mathrm{mmol}, 1.95 \mathrm{eq}$.), iodine (300 mg, 0.79 mmol, 0.12 eq.) and (2R,3S,4S,5R,6R)-2-(acetoxymethyl)-6-bromotetrahydro-2H-pyran3,4,5-triyl triacetate $(2.99 \mathrm{~g}, 1.18 \mathrm{mmol}, 1.1 \mathrm{eq}$.) with stirring. The mixture was heated at reflux for 2 days and then filtered over a pad of Celite. The filter pad was washed with chloroform $(3 \times 110 \mathrm{~mL})$ and the combined filtrate was washed sequentially with water, $0.1 \mathrm{M}$ sodium thiosulfate solution and finally with water. The organic solution was dried over sodium sulfate, filtered and evaporated to dryness to give a brown residue (3.0 g). The product was subjected to normal phase chromatographic purification $(80 \mathrm{~g}$ silica gel column) eluting with a gradient of $30 \%$ ethyl acetate in hexane to $100 \%$ ethyl acetate. The product fractions (eluting at $\sim 85 \%$ ethyl acetate) were concentrated to afford (2S,3R,4R,5S,6S)-2-(acetoxymethyl)-6-((6-(5-fluoro-2,4-dioxo-3,4-dihydropyrimidin-1(2H)- 
yl)-2,2-dimethyltetrahydrofuro[3,4-d][1,3]dioxol-4-yl)methoxy)tetrahydro-2H-pyran-3,4,5triyl triacetate as a solid white foam $(2.0 \mathrm{~g}, 32 \%$ yield $) \mathrm{m} / \mathrm{z}=632.56$ found $633.5[\mathrm{M}+\mathrm{H}]^{+}$. Step 3: To (2S,3R,4R,5S,6S)-2-(acetoxymethyl)-6-((6-(5-fluoro-2,4-dioxo-3,4dihydropyrimidin-1(2H)-yl)-2,2-dimethyltetrahydrofuro[3,4-d][1,3]dioxol-4yl)methoxy)tetrahydro-2H-pyran-3,4,5-triyl triacetate prepared above was added TFA (70 $\mathrm{mL})$ and water $(15 \mathrm{~mL})$ and the solution stirred at room temperature for 30 minutes. The solvent was concentrated to dryness and it was azeotroped with toluene $(4 \times 100 \mathrm{~mL})$ and toluene:methanol $(4 \times 100 \mathrm{~mL})$. The product was dried under high vacuum to give as (2S,3R,4R,5S,6S)-2-(acetoxymethyl)-6-((5-(5-fluoro-2,4-dioxo-3,4-dihydropyrimidin-1(2H)yl)-3,4-dihydroxytetrahydrofuran-2-yl)methoxy)tetrahydro-2H-pyran-3,4,5-triyl triacetate a white foam. $(2.0 \mathrm{~g}, 91 \%$ yield $) \mathrm{m} / \mathrm{z}=592.48$ found $593.3[\mathrm{M}+\mathrm{H}]^{+}$.

Step 4: (2S,3R,4R,5S,6S)-2-(Acetoxymethyl)-6-((5-(5-fluoro-2,4-dioxo-3,4dihydropyrimidin-1(2H)-yl)-3,4-dihydroxytetrahydrofuran-2-yl)methoxy)tetrahydro-2Hpyran-3,4,5-triyl triacetate $(1600 \mathrm{mg}, 2.7 \mathrm{mmol})$ was dissolved in methanol $(70 \mathrm{~mL})$. A solution of sodium methoxide $(0.61 \mathrm{~mL}, 25 \%$ in methanol, $2.85 \mathrm{mmol}, 1.05$ eq. $)$ was added slowly at ice bath temperature. The mixture was stirred at room temperature overnight, after which time LC/MS analysis showed the desired product. Resin $50 \mathrm{H}(1.0 \mathrm{~g})$ was added and the $\mathrm{pH}$ was checked ( 6.0). The solution was filtered and the filtrate was washed with methanol $(3 \times 100 \mathrm{~mL})$. The combined filtrate was concentrated to give an oil (1.3 g). The crude product was purified by HPLC to obtain the target compound 1((2R,3R,4S,5R)-3,4-dihydroxy-5-(((2R,3R,4S,5R,6R)-3,4,5-trihydroxy-6(hydroxymethyl)tetrahydro-2H-pyran-2-yl)oxy)methyl)tetrahydrofuran-2-yl)-5fluoropyrimidine-2,4(1H,3H)-dione, aka 5-fluorouridine-5'-O- $\beta$-D-galactopyranoside (788 $\mathrm{mg}, 68 \%$ yield) $\mathrm{m} / \mathrm{z}=424.1$ found $424.9[\mathrm{M}+\mathrm{H}]^{+} .{ }^{1} \mathrm{H}$ NMR $(500 \mathrm{MHz}$, Deuterium Oxide) $\delta$ $8.01(\mathrm{~d}, J=6.5 \mathrm{~Hz}, 1 \mathrm{H}), 5.81-5.77(\mathrm{~m}, 1 \mathrm{H}), 4.36(\mathrm{~d}, J=7.7 \mathrm{~Hz}, 1 \mathrm{H}), 4.27-4.10(\mathrm{~m}, 4 \mathrm{H})$, 
$3.83-3.79(\mathrm{~m}, 1 \mathrm{H}), 3.79-3.73(\mathrm{~m}, 1 \mathrm{H}), 3.72-3.65(\mathrm{~m}, 1 \mathrm{H}), 3.62(\mathrm{dd}, J=11.7,4.1 \mathrm{~Hz}$,

1H), $3.60-3.55(\mathrm{~m}, 1 \mathrm{H}), 3.53(\mathrm{dd}, J=9.9,3.4 \mathrm{~Hz}, 1 \mathrm{H}), 3.50-3.44(\mathrm{~m}, 1 \mathrm{H})$.

\section{Animals}

C57BL/6, ROSA26eYFP, CD-1 mice and FVB-Tg(CAG-luc,-GFP)L2G85Chco/J male mice were obtained from Jackson Laboratory. NOD/MrkBomTac-Prkdcscid female mice were obtained from Taconic Biosciences. Pax7CreER mouse were provided byDr Charles Keller, Oregon Health and Science University, Portland, OR, USA59.Tamoxifen injections for Cre recombinase activation were performed administering five doses (5 $\mathrm{mg}$ per mouse) every 2 days and waiting a minimum of 7 days before using the animals experimentally59. To control for tamoxifen injection toxicity, we injected all mice with tamoxifen. Mice were housed and maintained in the Veterinary Medical Unit at the Veterans Affairs Palo Alto Health Care Systems. Animal protocols were approved by the Administrative Panel on Laboratory Animal Care of Stanford University and of Molecular Medicine Research Institute (Sunnyvale, CA).

\section{Pharmacokinetic studies}

Concentrations of 5FU and 5FURGal were measured in plasma after IV dosing to male CD1 mice (N=3/dose group) at Pharmaron, Inc., Beijing, China. Compounds were formulated in 15\% EtOH/15\% PEG400/70\% saline and dosed at $5 \mathrm{mg} / \mathrm{kg}$ and $40 \mathrm{mg} / \mathrm{kg}$ respectively. Blood was sampled at 8 time points over $24 \mathrm{hrs}$, quenched with an equal volume of $25 \mathrm{mM}$ citrate buffer, and processed for plasma. Concentrations of compounds in plasma were determined using a specific LC-MS/MS method using a Shimazdu DGU20A5R HPLC, equipped with a $50 \times 3 \mathrm{~mm}$ Phenomenex Synergi $2.5 \mathrm{~mm}$ Polar-RP column and an API 5500 LC-MS/MS detector. Compounds were eluted using a mobile phase 
gradient comprising water/MeOH/0.1\% formic acid, and concentrations determined relative to calibration standards. Pharmacokinetics of 5FU and 5FURGal were calculated using WinNonlin (Phoenix, version 6.1).

\section{Voluntary running.}

Each subject was singly housed in cage with computer-controlled running wheels. All animals had access ad libitum to food, water and wheel running. Prior to the experimental period, subjects were given 3 days to acclimate to the cages and their surroundings. At day 3 , the experimental group had internal ablations performed on both legs as described in 'Ablation Model' section in 'Methods'. Mice were then returned to their cages with computer-controlled running wheels and allowed to run freely. Non-exercised controls were singly housed in cages with locked wheels. Cycles of 12-h day per night light were used in the rooms housing the mice and their cage bedding was changed once a week. Each cage wheel was attached to a counter motor that was then attached to data acquisition software that recorded the number of rotations that occurred during each 15 min interval for 2 weeks.

\section{Treadmill running.}

Before each mouse began its exercise programme, it was acclimated to the Exer $3 / 6$ treadmill (Columbus Instruments) for 3 days with a 10 min running period at $10 \mathrm{mmin}$ each day. At the start of each training regimen, the mice slowly acclimated to the speed of the treadmill by beginning at $7 \mathrm{~m} / \mathrm{min}$ and increasing the speed at $0.5 \mathrm{~m} / \mathrm{min}$ until a speed of $12 \mathrm{~m} / \mathrm{min}$ was reached. On the fourth day, mice were tested for endurance, running at a speed of $13 \mathrm{~m} /$ minute, with subsequent increments of $1 \mathrm{~m} /$ minute every minute. A low voltage was applied to a grate at the end of the treadmill to encourage the mice to 
continue running for the duration of the protocol. Mice were removed from the treadmill at the point of exhaustion, which was defined as staying 5 seconds on the electric grid $^{61}$.

\section{Grip test}

Mice were put on a BioSeb grip tester and their grip force measured for all four limbs. Mice were measured three-five times and the average was used to calculate grip strength. Values were normalized to mouse weight.

\section{Rotarod performance test}

Rotarod rotating cylinder (rod) (IITC Life Science Inc. Woodland Hills, CA, USA). The diameter of the rotating rod was $3.18 \mathrm{~cm}$. A first "trial run" was performed, prior to the experimental test. Once all animals were on the rod, the motor was turned on at a starting speed of four RPM, and the rod rotation was continuously accelerated until it reached a maximum speed of 40 RPM after $300 \mathrm{~s}$, which was the cut-off point to end the test. For each mouse, the trial was ended when the mouse fell from the rotating rod, or at the end of the cut-off point. The time it took the animal to fall from the rotating rod was recorded by a blinded experimenter.

\section{Y-Maze}

The Y-maze assesses spontaneous alternation as a readout for exploratory behavior and working memory in rodents. Our Y-maze consisted of 3 identical arms at $120^{\circ}$ angles from each other. Mice were introduced to the test room $10 \mathrm{~min}$ or more prior to testing. The test room, lighting, and position of the Y-maze were the same each time. The paper floor of the maze was replaced fresh and wiped clean for each trial. The mouse was carefully placed into the center of the Y-maze and allowed to explore freely for 5 minutes. Each complete 
entry into one arm of the Y-maze labeled A, B, or C was scored manually if all 4 limbs crossed the threshold. Upon completion, each mouse was returned carefully to their respective cage. Total entries and triads were recorded for each mouse to determine changes in treated vs control. Mice were tested before and after treatments and fold changes calculated accordingly.

\section{Morris Water Maze}

The mice were assessed post-injection with the Morris water maze for spatial learning and memory. The water maze was a steel circular pool $152 \mathrm{~cm}$ in diameter. It was filled with opaque white water at $21-22^{\circ} \mathrm{C}$. A black, square platform $(12.7 \mathrm{~cm} \times 12.7 \mathrm{~cm})$ was used for the training. Different shaped cutouts were placed just above the tank's rim in each quadrant to be used as visual cues. Prior to the training sessions, the mice were handled for the first 3 days within the same room as the water maze to acclimate them to the handlers and the room. On day 4-6, the mice were given 6 sessions to train with a platform that was raised $0.2 \mathrm{~cm}$ above the water level. During the visible platform training, both the platform and the drop location changed for each session. On day $7-11$, there were 10 sessions with a hidden platform by submerging it $1.5 \mathrm{~cm}$ below the water level. Throughout all the hidden platform sessions, the platform remained stationary while the drop location was changed. On day 12, a probe test without the platform was performed for 2 minutes. The tests were recorded and analyzed for the time spent within each quadrant.

\section{In Vivo Scoring}

Animals were scored for the onset of symptoms including dystonia, coat condition, ataxia, body condition, gait disorders. The investigator recording onset and severity of symptoms 
was blinded as to treatment groups. All symptoms were scored on a scale of 0 to 10 , with: a) 10 indicating no symptom; b) 5 indicating the symptom was detected but not consistently expressed; c) 0 indicating when the animal begins to express the symptom $100 \%$ of the time. For fur conditions, a scoring was assigned for each of the following conditions: 1) alopecia; 2) dermatitis; 3) loss of pigmentation; 4) lucidity; 5) organization.

\section{Ex vivo force production}

An 800A in vitro apparatus (Aurora Scientific) was used to perform the ex vivo testing of the TA muscles. The testing chamber, along with the Krebs solution (Sigma), was kept at $25{ }^{\circ} \mathrm{C}$ using an external water heater. To help ensure tissue viability, a 95\% O2, 5\% CO2 mixture was used to oxygenate the testing chamber along with a petri dish containing Krebs solution. Mice were killed and the lower limbs excised from the ankle to B0.5 inches above the patellar tendon to ensure the entire TA muscle was removed. The legs were then pinned down in the petri dish coated with an elastomer (Sylgard-184, Dow Corning). The TA muscles were carefully dissected from the legs under a microscope and held under tension until attached to the force transducer lever. The muscle was attached to the force transducer lever by directly tying a knot around the top of the patellar tendon. The TA muscle was then attached to the force transducer lever and the bottom of the testing apparatus to secure the TA muscle in place. The testing chamber was raised around it to immerse the muscle in Krebs solution. A similar testing procedure to the in vivo protocol was used ex vivo except no voltage recruitment testing was performed. Imaging was performed in bind: the investigators performing the imaging did not know the identity of the experimental conditions for the transplanted cells. 


\section{Bioluminescence imaging}

Bioluminescent imaging was performed using the Xenogen IVIS-Spectrum System (Caliper Life Sciences). Mice were anaesthetized using $2 \%$ isoflurane at a flow rate of $2.5 \mathrm{I}$ min_1 (n.4). Intraperitoneal injection of D-Luciferin (50 mg ml_1, Biosynth International Inc.) or LUCGal ( $80 \mathrm{mg} / \mathrm{Kg}$, Promega) dissolved in sterile PBS was administered. Immediately following the injection, mice were imaged for $30 \mathrm{~s}$ at maximum sensitivity (fstop 1) at the highest resolution (small binning). Every minute a $30 \mathrm{~s}$ exposure was taken, until the peak intensity of the bioluminescent signal began to diminish. Each image was saved for subsequent analysis. Imaging was performed in bind: the investigators performing the imaging did not know the identity of the experimental conditions for the transplanted cells.

\section{Bioluminescence image analysis}

Analysis of each image was performed using Living Image Software, version 4.0 (Caliper Life Sciences). A manually-generated circle was placed on top of the region of interest and resized to completely surround the limb or the specified region on the recipient mouse. Similarly, a background region of interest was placed on a region of a mouse outside the transplanted leg.

\section{Histology}

TA muscles were fixed for $5 \mathrm{~h}$ using $0.5 \%$ electron-microscopy-grade paraformaldehyde and subsequently transferred to $20 \%$ sucrose overnight. Muscles were then frozen in optimal cutting temperature, cryosectioned at a thickness of $10 \mathrm{~mm}$ and stained. For colorimetric staining with Hematoxylin and Eosin (Sigma) or Gomorri Trichrome (RichardAllan Scientific) samples were processed according to the manufacturer's recommended protocols. For SA- $\beta$-Gal, stock solution was prepared using Corp X-GAL (Fisher Scientific) 
and staining was performed in combination with Feulgen Stain Kit (American Mastertech) for nuclear staining.

\section{Immunostaining}

A $1 \mathrm{~h}$ blocking step with $20 \%$ donkey serum/0.3\% Triton in PBS was used to prevent unwanted primary antibody binding for all samples. Primary antibodies were applied and allowed to incubate overnight at $4^{\circ} \mathrm{C}$ in $20 \%$ donkey serum $/ 0.3 \%$ Triton in PBS. After four washes with $0.3 \%$ PBST, fluorescently conjugated secondary antibodies were added and incubated at room temperature for $1 \mathrm{~h}$ in $0.3 \%$ PBST. After three additional rinses each slide was mounted using Fluoview mounting media. Photographs were acquired using standard fluorescent microscope and a CCD camera. Volocity imaging software. Was used to adjust excitation and emission filters and came with AlexaFluor filter settings, which were used whenever possible. All exposure times were optimized during the first round of imaging and then kept constant through all subsequent imaging. The immunoreactivity was quantified as the percent area covered by ImageJ (ref). For each staining, a total of three hippocampal brain sections per mouse were analyzed.

\section{Antibodies.}

The following antibodies were used in this study. The source of each antibody is indicated. Mouse: GFP (Invitrogen, \#A11122, 1:250); Collagen I (Cedarlane Labs, \#CL50151AP, 1:200); Laminin (Millipore, \#MAB1903, 1:750); ADD CD68 (AbD Serotec, 1:50), and Iba1 (Wako Chemicals, USA, 1:1250).

\section{Muscle Stem Cell Isolation and purification}

Muscles were harvested from hind limbs and mechanically dissociated to yield a fragmented muscle suspension using a gentleMACS dissociator (Miltenyl Biotec). This 
was followed by a 90 min digestion in a Collagenase II-Ham's F10 solution (500 units per $\mathrm{ml}$; Invitrogen). After washing, a second digestion was performed for 30 min with Collagenase II (100 units per $\mathrm{ml}$ ) and Dispase (2 units per $\mathrm{ml}$; Invitrogen). For Pax7CreER/ROSA26eYFP mice, MuSCs were directly sorted by endogenous eYFP protein expression. Cell sorting was performed on calibrated BD-FACS Aria II or BD FACSAria III flow cytometers equipped with 488-nm, 633-nm and 405-nm lasers to obtain the MuSC population. A small fraction of sorted cells was plated and stained for Pax7 and MyoD to assess the purity of the sorted population

\section{Myogenic colony-forming cell assay for MuSCs}

Single treated and control MuSCs were deposited into wells of collagen- and laminincoated plates at 1 cell per well by BD FACSAria III flow cytometer. Collagen/laminin coating was accomplished by overnight incubation of the plates rocking at $4^{\circ} \mathrm{C}$ with a $1: 1$ mixture of laminin $(10 \mu \mathrm{g} / \mathrm{ml}$ ThermoFisher $23017-015)$ and collagen $(10 \mu \mathrm{g} / \mathrm{ml}$ Sigma C8919) in PBS. Coated wells were washed three times with PBS before use. The cells were cultured in grow media, F10 medium supplemented with $20 \%$ horse serum and 5 ng/ml basic fibroblast growth factor (bFGF; PeproTech 100-18B). After 6 days of culture, plates were fixed with 4\% paraformaldehyde (Electron Microscopy Services 15710), stained with DAPI (Invitrogen D1306), and scored by microscopy to determine the number of myogenic colony-forming cells (MCFCs), defined by wells that contained at least 8 cells.

\section{EDU staining}

Staining was done according to the manufacturer protocol using the Click-iT EdU kit. Cells were label with Edu after switching to growth media. Cells were allowed to grow one day before fixation with $4 \%$ paraformaldehyde and permeabilization with $0.5 \%$ Triton $X-100$ in 
PBST. Cells were the incubated in Click-It reaction cocktail for 30 min before washing in PBS and imaging.

\section{Complete Blood Counts}

Mice were anesthetized using isoflurane and blood was collected via cardiac puncture using a $1 \mathrm{~mL}$ syringe with a 25 gauge needle. Blood was transferred immediately to a K2EDTA microtainer tube, inverted 8 to 10 times and placed it on a rocker at room temperature. Blood was tested on a VETSCAN HM5 Hematology Analyzer running a software VetScan HM5 v2.3.

\section{Quantitative PCR}

For RNA extraction, samples were collected in RNAlater solution (Sigma-Aldrich, USA). Total RNA was isolated by using Quick-RNA ${ }^{\mathrm{TM}}$ Miniprep Plus Kit (Zymo Research). For mRNA analysis, isolated RNA was reverse transcribed using the High-Capacity cDNA Reverse Transcription Kit (Life Technologies) and qPCR was carried out on QuantSudio 5 Instrument (Applied Biosystem) using PowerUp ${ }^{\text {TM }}$ SYBR® Green (Thermo Fisher Scientific) and gene-specific primers (Invitrogen). Relative quantification of gene expression was calculated by using the comparative CT method. PCR primer pairs used were: Cdkn2a Fwd: 5'-CCCAACGCCCCGAACT-3'; Rev: 5'GCAGAAGAGCTGCTACGTGAA-3'.

\section{Image analysis}

All other analyses were performed using Image $\mathrm{J}$ or Volocity software and manually counting fibres using the free draw tool and also counting the number of nuclei and CSA of myofibers. 


\section{Statistical analysis}

Unless otherwise noted, all statistical analyses were performed using GraphPad Prism 5 (GraphPad Software). For statistical analysis, t-tests were used. All error bars represent s.e.m.; ${ }^{*}$ Po0.05; ${ }^{* *}$ Po0.001; ${ }^{* * * P o 0.0001 . ~}$

\section{Data availability}

The data that support the findings of this study are available from the corresponding author upon reasonable request. 


\section{References}

1. Organization, W. H. WHO clinical consortium on healthy ageing: topic focus: frailty and intrinsic capacity: report of consortium meeting, 1-2 December 2016 in Geneva, Switzerland. (2017).

2. Fairhall, N. et al. Treating frailty-a practical guide. BMC Med. 9, 83 (2011).

3. Fried, L. P., Darer, J. \& Walston, J. Frailty. in Geriatric Medicine 1067-1076 (SpringerVerlag, 2003). doi:10.1007/0-387-22621-4_74.

4. Clegg, A., Young, J., lliffe, S., Rikkert, M. O. \& Rockwood, K. Frailty in elderly people. The Lancet 381, 752-762 (2013).

5. Eeles, E. M. P., White, S. V., O'Mahony, S. M., Bayer, A. J. \& Hubbard, R. E. The impact of frailty and delirium on mortality in older inpatients. Age Ageing 41, 412-416 (2012).

6. Song, X., Mitnitski, A. \& Rockwood, K. Prevalence and 10-Year Outcomes of Frailty in Older Adults in Relation to Deficit Accumulation: FRAILTY PREVALENCE AND OUTCOME. J. Am. Geriatr. Soc. 58, 681-687 (2010).

7. Fried, L. P. et al. Frailty in Older Adults: Evidence for a Phenotype. J. Gerontol. A. Biol. Sci. Med. Sci. 56, M146-M157 (2001).

8. Priority Areas for National Action: Transforming Health Care Quality. 10593 (National Academies Press, 2003). doi:10.17226/10593.

9. Hayflick, L. The limited in vitro lifetime of human diploid cell strains. Exp. Cell Res. 37, $614-636$ (1965).

10. Lopes-Paciencia, S. et al. The senescence-associated secretory phenotype and its regulation. Cytokine 117, 15-22 (2019).

11. Childs, B. G., Durik, M., Baker, D. J. \& van Deursen, J. M. Cellular senescence in aging and age-related disease: from mechanisms to therapy. Nat. Med. 21, 1424-1435 (2015). 
12. Xu, M. et al. Senolytics improve physical function and increase lifespan in old age. Nat. Med. 24, 1246-1256 (2018).

13. Childs, B. G. et al. Senescent intimal foam cells are deleterious at all stages of atherosclerosis. Science 354, 472-477 (2016).

14. Baker, D. J. et al. Clearance of p16 Ink4a -positive senescent cells delays ageingassociated disorders. Nature 479, 232-236 (2011).

15. Chang, J. et al. Clearance of senescent cells by ABT263 rejuvenates aged hematopoietic stem cells in mice. Nat. Med. 22, 78-83 (2016).

16. Baker, D. J. et al. Naturally occurring p16(Ink4a)-positive cells shorten healthy lifespan. Nature 530, 184-189 (2016).

17. Baar, M. P. et al. Targeted Apoptosis of Senescent Cells Restores Tissue Homeostasis in Response to Chemotoxicity and Aging. Cell 169, 132-147.e16 (2017).

18. Zhu, Y. et al. The Achilles' heel of senescent cells: from transcriptome to senolytic drugs. Aging Cell 14, 644-658 (2015).

19. Kim, E.-C. \& Kim, J.-R. Senotherapeutics: emerging strategy for healthy aging and age-related disease. BMB Rep. 52, 47-55 (2019).

20. Roos, C. M. et al. Chronic senolytic treatment alleviates established vasomotor dysfunction in aged or atherosclerotic mice. Aging Cell 15, 973-977 (2016).

21. Farr, J. N. et al. Targeting cellular senescence prevents age-related bone loss in mice. Nat. Med. 23, 1072-1079 (2017).

22. Schafer, M. J. et al. Cellular senescence mediates fibrotic pulmonary disease. Nat. Commun. 8, 14532 (2017).

23. Ogrodnik, M. et al. Cellular senescence drives age-dependent hepatic steatosis. Nat. Commun. 8, 15691 (2017).

24. Bussian, T. J. et al. Clearance of senescent glial cells prevents tau-dependent pathology and cognitive decline. Nature 562, 578-582 (2018). 
25. Hwang, H. V., Tran, D. T., Rebuffatti, M. N., Li, C.-S. \& Knowlton, A. A. Investigation of quercetin and hyperoside as senolytics in adult human endothelial cells. PloS One 13, e0190374 (2018)

26. Kovacovicova, K. et al. Senolytic Cocktail Dasatinib+Quercetin (D+Q) Does Not Enhance the Efficacy of Senescence-Inducing Chemotherapy in Liver Cancer. Front. Oncol. 8, (2018).

27. Leverson, J. D. et al. Exploiting selective BCL-2 family inhibitors to dissect cell survival dependencies and define improved strategies for cancer therapy. Sci. Transl. Med. 7, 279ra40 (2015).

28. Li, Q. et al. Efficacy and Safety of Bcl-2 Inhibitor Venetoclax in Hematological Malignancy: A Systematic Review and Meta-Analysis of Clinical Trials. Front. Pharmacol. 10, (2019).

29. de Magalhães, J. P. \& Passos, J. F. Stress, cell senescence and organismal ageing. Mech. Ageing Dev. 170, 2-9 (2018).

30. Salmon, A. B. About-face on the metabolic side effects of rapamycin. Oncotarget 6 , 2585-2586 (2015).

31. Kirkland, J. L., Tchkonia, T., Zhu, Y., Niedernhofer, L. J. \& Robbins, P. D. The Clinical Potential of Senolytic Drugs. J. Am. Geriatr. Soc. 65, 2297-2301 (2017).

32. Cho, S. \& Hwang, E. S. Status of mTOR Activity May Phenotypically Differentiate Senescence and Quiescence. Mol. Cells 33, 597-604 (2012).

33. Hwang, E. S., Yoon, G. \& Kang, H. T. A comparative analysis of the cell biology of senescence and aging. Cell. Mol. Life Sci. CMLS 66, 2503-2524 (2009).

34. Dimri, G. P. et al. A biomarker that identifies senescent human cells in culture and in aging skin in vivo. Proc. Natl. Acad. Sci. 92, 9363-9367 (1995).

35. Pendergrass, W. R. et al. Cellular proliferation potential during aging and caloric restriction in rhesus monkeys (Macaca mulatta). J. Cell. Physiol. 180, 123-130 (1999). 
36. Melk, A. et al. Cell senescence in rat kidneys in vivo increases with growth and age despite lack of telomere shortening. Kidney Int. 63, 2134-2143 (2003).

37. Guerrero, A. et al. Galactose-modified duocarmycin prodrugs as senolytics. Aging Cell 19, e13133 (2020)

38. Cai, Y. et al. Elimination of senescent cells by $\beta$-galactosidase-targeted prodrug attenuates inflammation and restores physical function in aged mice. Cell Res. 30, 574-589 (2020).

39. González-Gualda, E. et al. Galacto-conjugation of Navitoclax as an efficient strategy to increase senolytic specificity and reduce platelet toxicity. Aging Cell 19, e13142 (2020).

40. Parker, J. B. \& Stivers, J. T. Dynamics of Uracil and 5-Fluorouracil in DNA. Biochemistry 50, 612-617 (2011).

41. Zombeck, J. A., Fey, E. G., Lyng, G. D. \& Sonis, S. T. A Clinically Translatable Mouse Model for Chemotherapy-Related Fatigue. Comp. Med. 63, 491-497 (2013).

42. Hurria, A., Jones, L. \& Muss, H. B. Cancer Treatment as an Accelerated Aging Process: Assessment, Biomarkers, and Interventions. Am. Soc. Clin. Oncol. Educ. Book e516-e522 (2016) doi:10.1200/EDBK_156160.

43. da Silva, V. D. et al. Association between frailty and the combination of physical activity level and sedentary behavior in older adults. BMC Public Health 19, 709 (2019).

44. Miyamura, K. et al. Frailty syndrome and cognitive impairment in older adults: systematic review of the literature. Rev. Lat. Am. Enfermagem 27,.

45. Boyle, P. A., Buchman, A. S., Wilson, R. S., Leurgans, S. E. \& Bennett, D. A. Physical Frailty Is Associated with Incident Mild Cognitive Impairment in Community-Based Older Persons. J. Am. Geriatr. Soc. 58, 248-255 (2010).

46. Wolf, A., Bauer, B., Abner, E. L., Ashkenazy-Frolinger, T. \& Hartz, A. M. S. A Comprehensive Behavioral Test Battery to Assess Learning and Memory in 129S6/Tg2576 Mice. PLOS ONE 11, e0147733 (2016). 
47. Spittau, B. Aging Microglia-Phenotypes, Functions and Implications for Age-Related Neurodegenerative Diseases. Front. Aging Neurosci. 9, (2017).

48. Larsson, L. et al. Sarcopenia: Aging-Related Loss of Muscle Mass and Function. Physiol. Rev. 99, 427-511 (2018).

49. Alway, S. E., Myers, M. J. \& Mohamed, J. S. Regulation of Satellite Cell Function in Sarcopenia. Front. Aging Neurosci. 6, (2014).

50. Sarkar, T. J. et al. Transient non-integrative expression of nuclear reprogramming factors promotes multifaceted amelioration of aging in human cells. Nat. Commun. 11, 1545 (2020).

51. Ritt, M., Ritt, J. I., Sieber, C. C. \& Gaßmann, K.-G. Comparing the predictive accuracy of frailty, comorbidity, and disability for mortality: a 1-year follow-up in patients hospitalized in geriatric wards. Clin. Interv. Aging 12, 293-304 (2017).

52. Aubertin-Leheudre, M., Woods, A. J., Anton, S., Cohen, R. \& Pahor, M. Frailty Clinical Phenotype: A Physical and Cognitive Point of View. Frailty Pathophysiol. Phenotype Patient Care 83, 55-64 (2015).

53. Pignolo, R. J., Passos, J. F., Khosla, S., Tchkonia, T. \& Kirkland, J. L. Reducing Senescent Cell Burden in Aging and Disease. Trends Mol. Med. 26, 630-638 (2020).

54. Reuben, D. B., Judd-Hamilton, L., Harris, T. B. \& Seeman, T. E. The Associations Between Physical Activity and Inflammatory Markers in High-Functioning Older Persons: MacArthur Studies of Successful Aging. J. Am. Geriatr. Soc. 51, 1125-1130 (2003).

55. Kane, A. E. \& Sinclair, D. A. Frailty biomarkers in humans and rodents: Current approaches and future advances. Mech. Ageing Dev. 180, 117-128 (2019).

56. Beyer, I. et al. Inflammation-related muscle weakness and fatigue in geriatric patients. Exp. Gerontol. 47, 52-59 (2012).

57. Lu, Y. et al. Inflammatory and immune markers associated with physical frailty 
syndrome: findings from Singapore longitudinal aging studies. Oncotarget 7, 2878328795 (2016).

58. Kao, T.-W. et al. Examining how p16 INK4a expression levels are linked to handgrip strength in the elderly. Sci. Rep. 6, 31905 (2016).

59. Justice, J. N. et al. Cellular Senescence Biomarker p16INK4a+ Cell Burden in Thigh Adipose is Associated With Poor Physical Function in Older Women. J. Gerontol. A. Biol. Sci. Med. Sci. 73, 939-945 (2018).

60. Kirkland, J. L. AFFIRM: A Phase 2 Randomized, Placebo-Controlled Study of Alleviation by Fisetin of Frailty, Inflammation, and Related Measures in Older Women. https://clinicaltrials.gov/ct2/show/NCT03430037 (2020).

61. Laker, R. C. et al. Ampk phosphorylation of Ulk1 is required for targeting of mitochondria to lysosomes in exercise-induced mitophagy. Nat. Commun. 8, (2017).

\section{Acknowledgments}

We thank the members of the Rubedo Life Sciences, Rando and Wyss-Coray laboratories for comments and discussions; we thank Julian Klein for technical support. This work was supported by Rubedo Life Sciences, the Glenn Foundation for Medical Research and the CalPoly funding Award \# TB1-01175 supporting Rubedo Life Sciences.

\section{Author Contributions}

M.Q., J.L. and T.A.R. conceived, designed and/or supervised the experiments reported. L.D., P.P., C.T., A.H., A.D.M., H.I. and A.C.V. performed the in vivo experiments and analysis. C.T., I.J. and B.P. performed the in vitro experiments and analysis. P.P. and N.C. performed the histology, imaging and analysis. L.D., P.P., C.T., B.P., A.H., I.J., N.C., A.C.V., A.D.M., J.R.J., M.A.G., J.L., T.W., T.A.R. and M.Q. analyzed data, provided 
technical or scientific guidance. M.Q., M.A.G., J.R.J., A.C.V. and L.D. wrote the manuscript with inputs from all the authors.

\section{Competing Financial Interest}

The authors L.D., P.P., B.P., A.H., N.C., A.C.V., J.R.J., M.A.G., and M.Q. are either current or former employees and shareholders of Rubedo Life Sciences. M.Q. and M.A.G. are co-founders of Rubedo Life Sciences. 


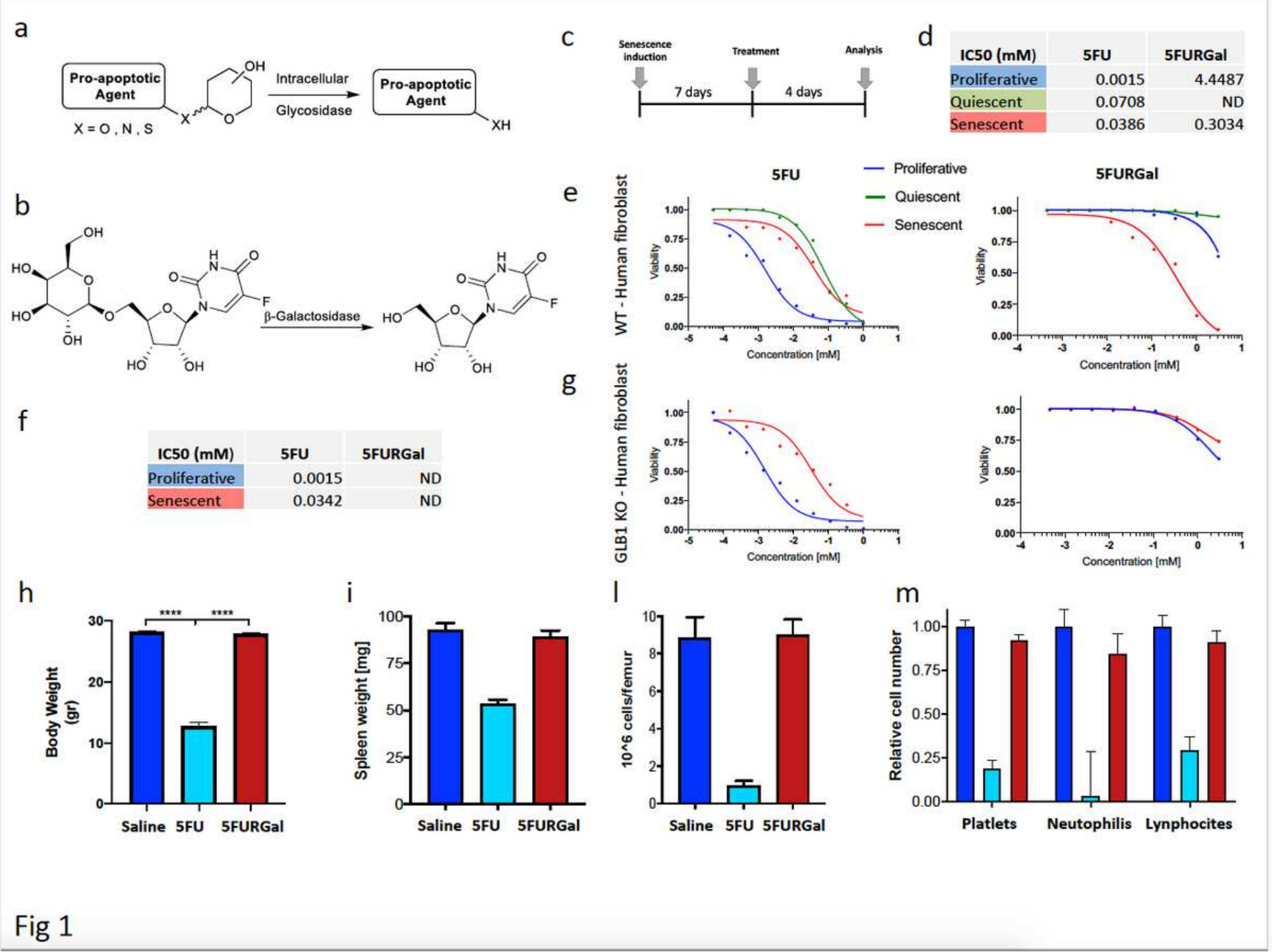

Figure 1

5FURGal targets senescence without affecting proliferation and quiescence in vitro and toxicity in vivo. a. Schematic model of the strategy employed to generate prodrug strategy. b. Molecular structure of the prodrug 5FURGal and the parent drug 5FU. c. experimental protocol employed to test senolytic efficacy in vitro. d. IC50 values reported for human fibroblasts cell line cultured in the following conditions: 1 ) proliferative; 2) senescence; 3) quiescence with either 5FU or 5FURGal. e. Quantification of cell viability as indicated in panel (e) at different concentrations of either 5-FUR (left panel) or 5FURGal (right panel). $\mathrm{f}$. IC50 values reported for human fibroblasts cell line KO for the hydrolase Galactosidase cultured in the following conditions: 1) proliferative; 2) senescence with either 5-FUR or 5FURGal. g. Quantification of cell viability as indicated in panel ( $f$ ) at different concentrations of either 5-FUR (left panel) or 5FURGal (right panel). h-m. Tolerability study. Three separate cohorts of WT C57/B6 mice received two IP injections separated by two days of respectively: 1) saline; 2) 5FU; or 3) 5-FUR-Gal. At the terminal time point animal body weight was measured ( $h$ ) before isolating and quantifying: 1) spleen weight (i); Bone marrow (l); 
hematopoietic cells $(\mathrm{m}) . \mathrm{n}=5$. Data are \pm s.e.m. For statistical analysis, t-tests were used. ${ }^{* \star *} \mathrm{P}<0.0001$, $\star \star \star \star P<0.00001$.

a

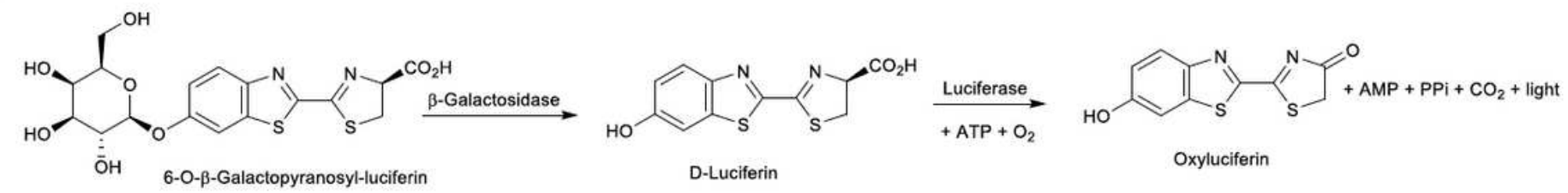

b

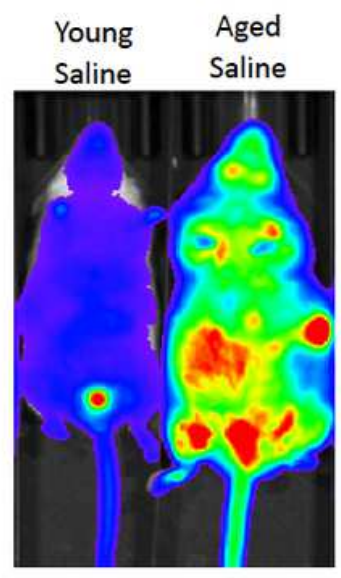

$$
\text { Young }
$$

C
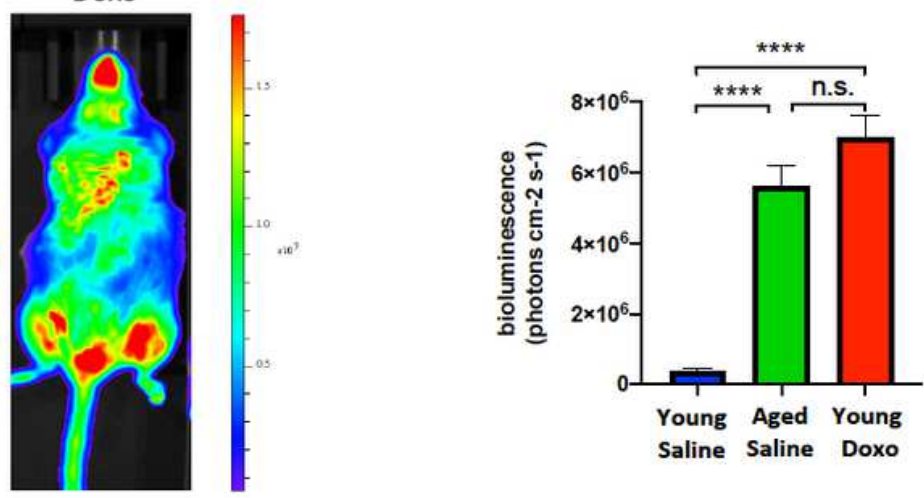

d

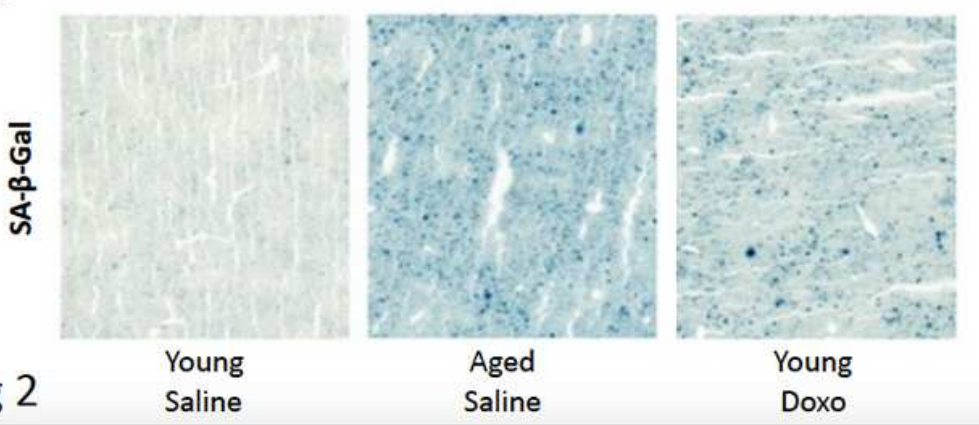

e

f
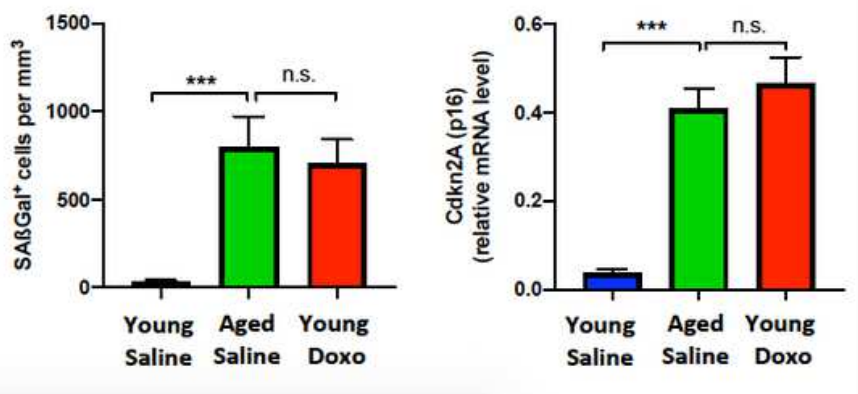

Figure 2

Prodrug based bioluminescence shows that chemotoxicity and aging are associated with senescence burden. a. Molecular structures and enzymatic reaction steps of the prodrug Galacto-Luciferine into Oxyluciferin. b. Representative images of bioluminescence measured from mice constitutively expressing Luciferase and injected with Galacto-luciferine prodrug at: 1) 4 months of age (Young); 2$) 24$ months of age (Aged); or 4 months of age treated with Doxorubicin. Mice received one IP injection of Galactoluciferine and immediately imagedc. $c$. Quantified results of non-invasive bioluminescence imaging ( $\mathrm{n}=$ 6). d. Representative SA- $\beta-G$ al images from cross-sections of liver tissue after receiving treatments with saline or doxorubicin (Doxo) at different ages. e. Quantification of SA- $\beta$-Gal positive cells in liver tissue (n = 6). f. Quantification by qPCR of Cdkn2A gene expression from liver tissue $(n=6)$. Data are $\pm s$.e.m. For statistical analysis, t-tests were used. ${ }^{*} * * P<0.0001,{ }^{*} * \star * P<0.00001$. 
a

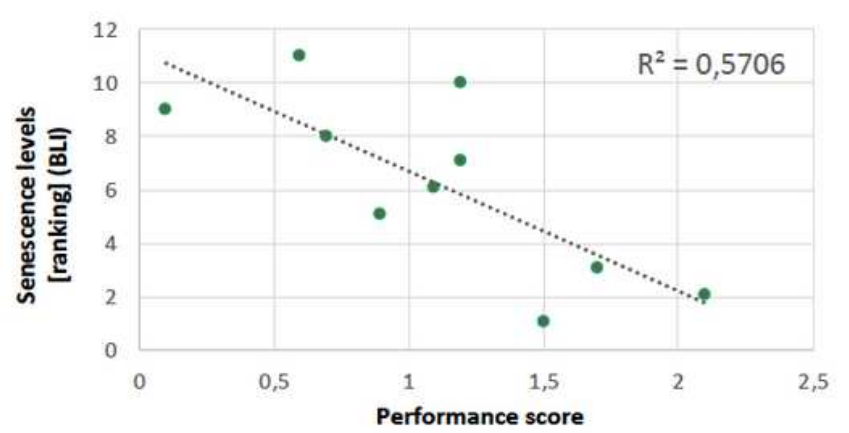

d

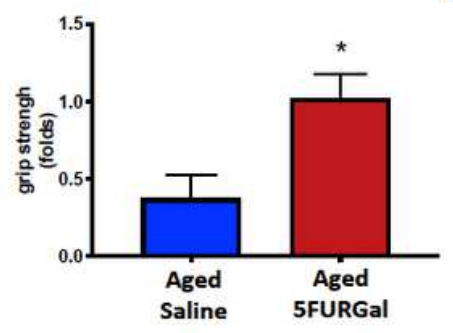

e

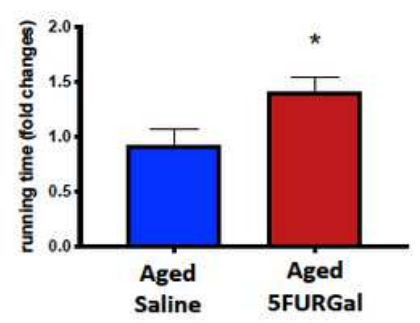

h

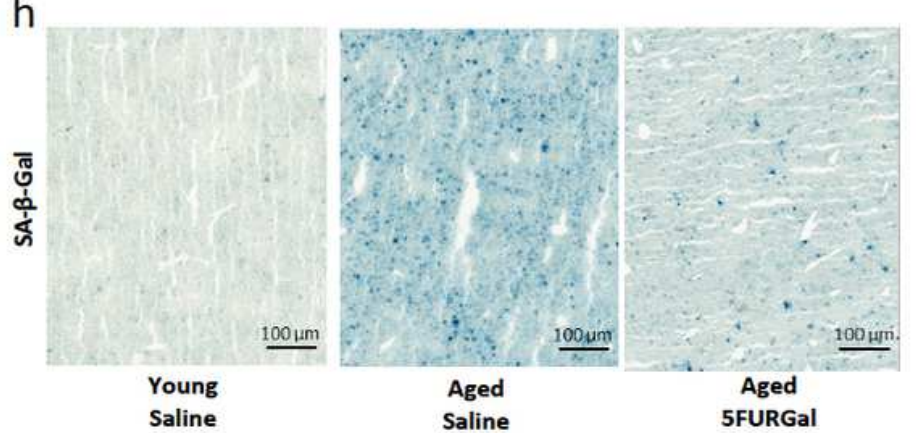

b

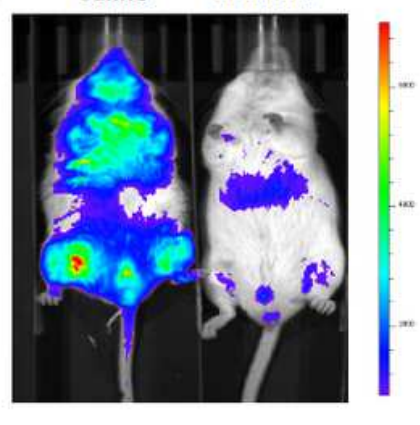

f

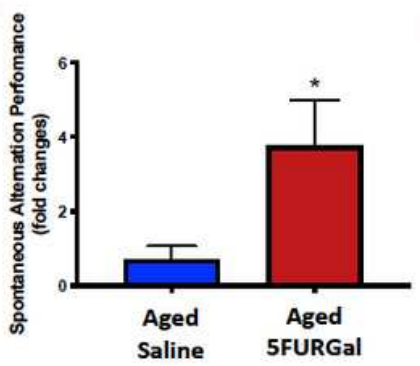

i

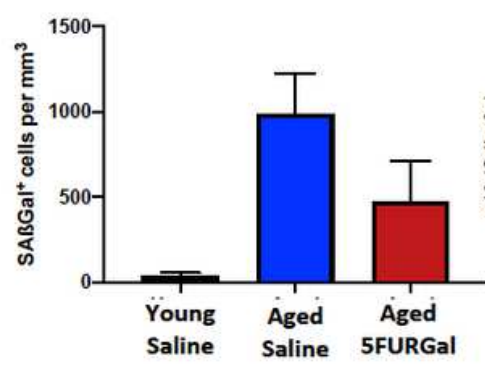

C

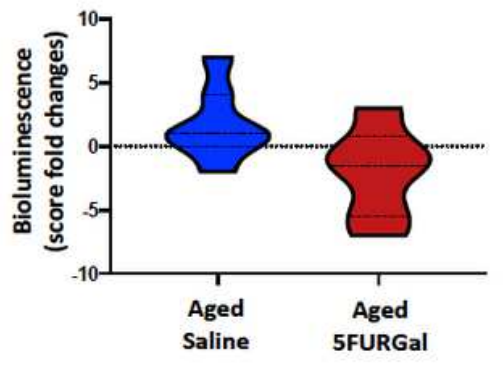

g

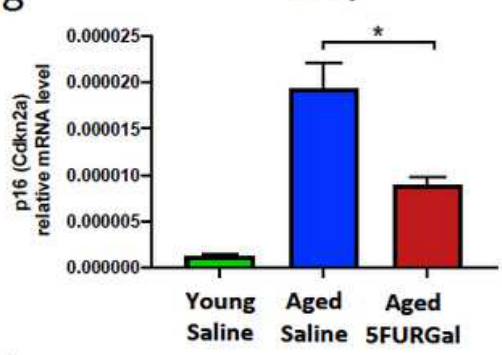

I

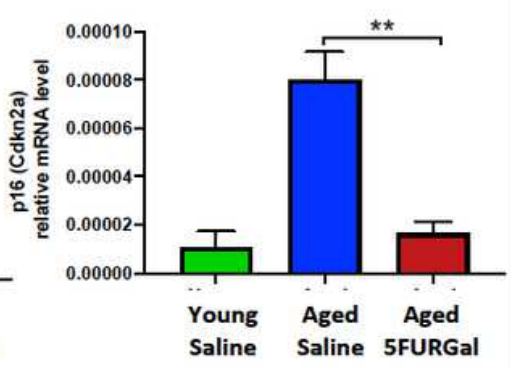

Fig 3

\section{Figure 3}

Prodrug senolytic treatment reduces senescence and improves physiological functions in aged mice. a. Correlation between bioluminescence and performance. Bioluminescence in mice expressing Luciferase of 24 months of age were measured following injection of Galacto-Luciferin prodrug. Animals were then assessed for physiological functions. Specifically, measurements for grip test for limb voluntary force measurement, endurance on treadmill, balance on rotarod test, cognitive functions on $Y$ maze test were collected and combined in a performance score. Each dot represents a mouse. b. Representative images of bioluminescence measured from mice constitutively expressing Luciferase and injected with Galactoluciferine prodrug in mice of 24 months of age (aged) treated with saline or 5-FUR-Gal prodrug. C. Quantified results of non-invasive bioluminescence imaging $(n=6)$. Functional assessment in aged mice treated with 5- FUR-Gal prodrug. Following bioluminescence imaging shown in (b and c), the same mice were assessed for: (d) voluntary limb force on grip strength test; (e) endurance on a treadmill test ; (f) spatial cognitive memory on a $Y$ maze test. Mice were assessed before and after receiving treatment with either saline or 5-FUR-Gal. g. Quantification by qPCR of Cdkn2A gene expression from kidney tissue $(n=$ 6). h. Representative SA- $\beta$-Gal images from cross-sections of liver tissue after receiving treatments with 
saline or 5-FURGal at different ages. i. Quantification of SA- $\beta$-Gal positive cells in liver tissue $(n=6)$. I. Quantification by qPCR of Cdkn2A gene expression from liver tissue $(n=6)$. Data are \pm s.e.m. For statistical analysis, t-tests were used. ${ }^{*} \mathrm{P}<0.01,{ }^{\star} * \mathrm{P}<0.001$.

a

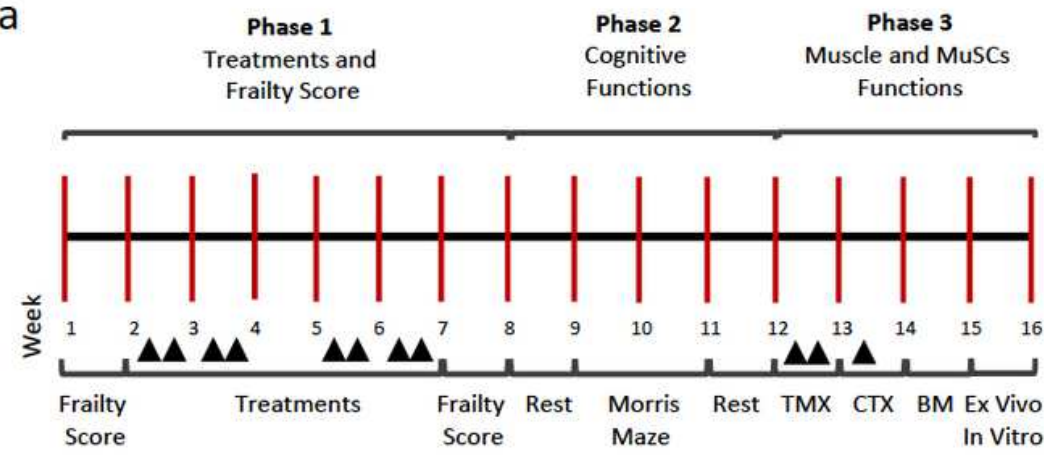

C

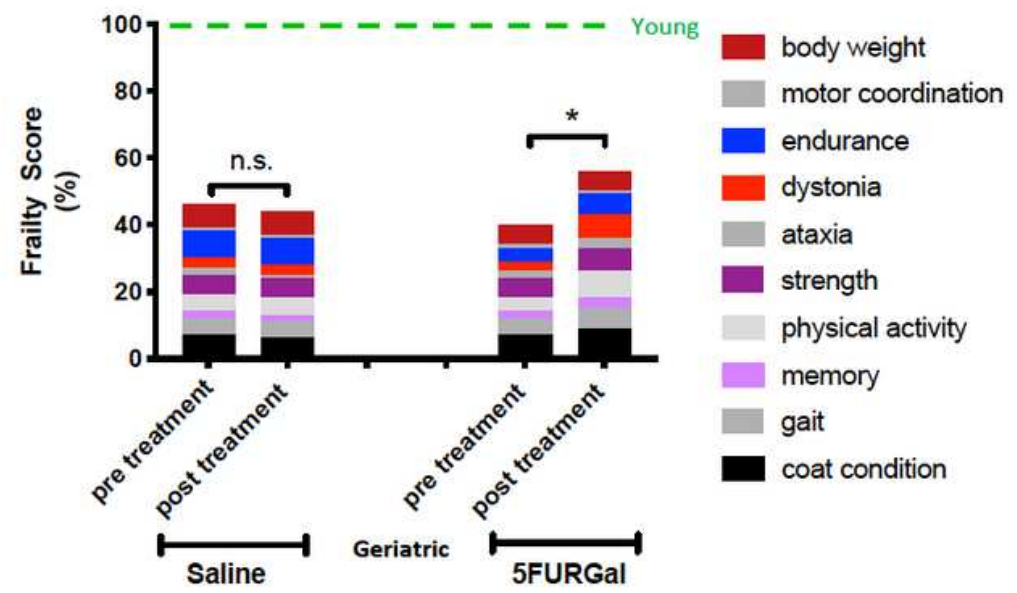

b

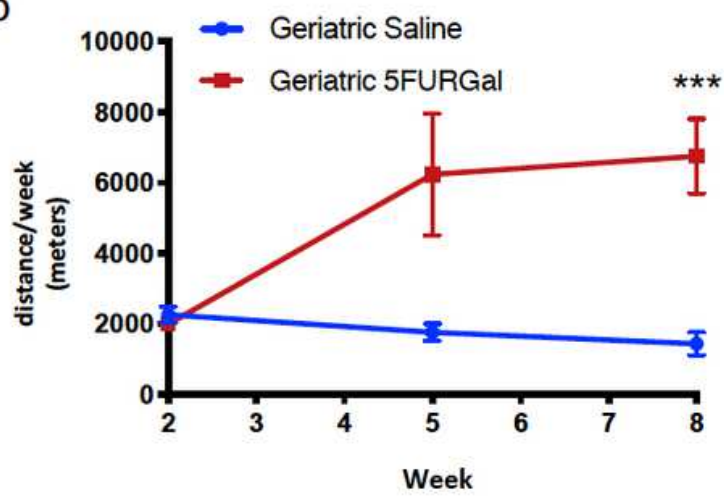

d

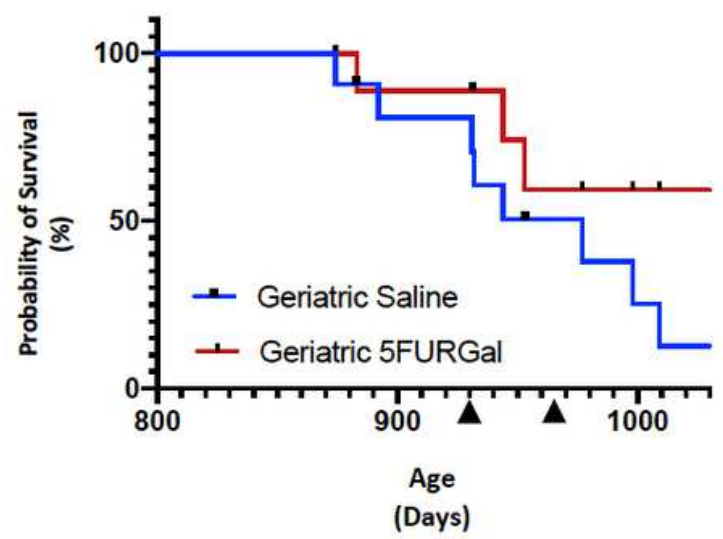

Fig 4

\section{Figure 4}

Prodrug senolytic treatment reverts frailty in geriatric mice. a. Schematic of the experimental design for the frailty study. Phase 1: Young or Geriatric mice were individually housed in cages with a computer controlled running wheel and acclimated for one week before being assessed for their frailty score baseline. Geriatric mice were then divided in two cohorts. One cohort of geriatric mice and the cohort of young mice received treatments with saline. The second cohort of geriatric mice received treatment with 5FURGal. All animals received one IP injection with either saline or 5FURGal twice per week for two weeks, followed by a week of rest and another two-week of treatments. At the end of the treatments, all animals were assessed again for the frailty score. Phase 2: Following a week of rest, all mice were subjected for two weeks to Morris water maze to assess cognitive functions. Phase 3: following a week of rest, all mice received two injections of tamoxifen (TMX) separated by three days to induce recombination of the fluorescent protein YFP in MuSCs expressing Pax7. After 10 days all mice received an injection of 
Cardiotoxin (CTX) in both TA muscles to induce skeletal muscle injury, activation of quiescent MuSCs and the tissue regenerative process. After 10 days all animals were sacrificed, TA muscles immediately assessed for biomechanics (BM) to measure contractile properties. were then MuSCs isolated for in vitro studies or histological assessments. Black arrowheads indicate time of injections. b. Quantification of spontaneous activity measured with running wheels rotations. Total distance run is quantified for geriatric mice: 1) before initiating treatments (day 3); 2) three weeks of treatments (Day 21);3) 5 weeks after treatments (day 35). c. Frailty Score. Young and geriatric mice were assessed for Frailty score before and after treatments with saline (young and geriatric mice) or 5FURGal (geriatric mice). Ten different parameters were measured, each weighing for up to $10 \%$ for a total of $100 \%$ based on young mice scoring (green broken line). d. Survival of geriatic mice during treatments. Geriatric mice were monitored during treatments with either saline or 5FURGal and their time of death recorded. Black arrowheads indicate beginning and end of treatments. Data are \pm s.e.m. For statistical analysis, t-tests were used. ${ }^{*} P<0.01,{ }^{*} * \mathrm{P}<0.0001 . n=7$.

a
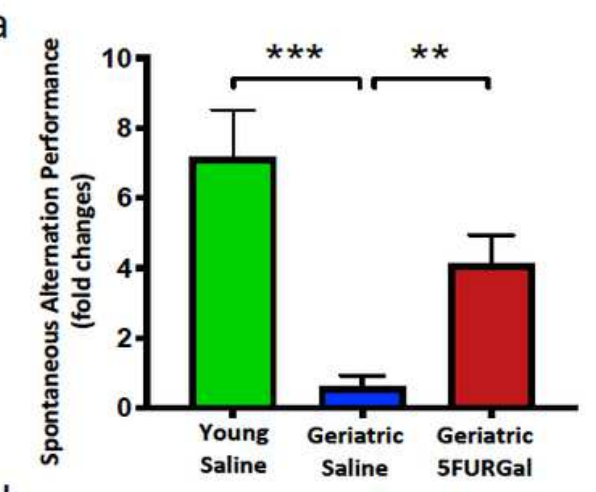

d

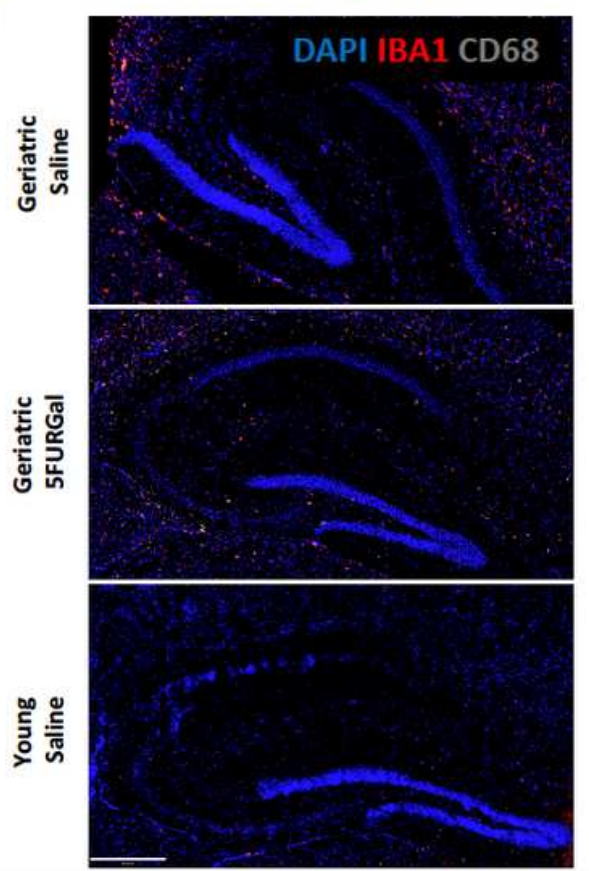

b

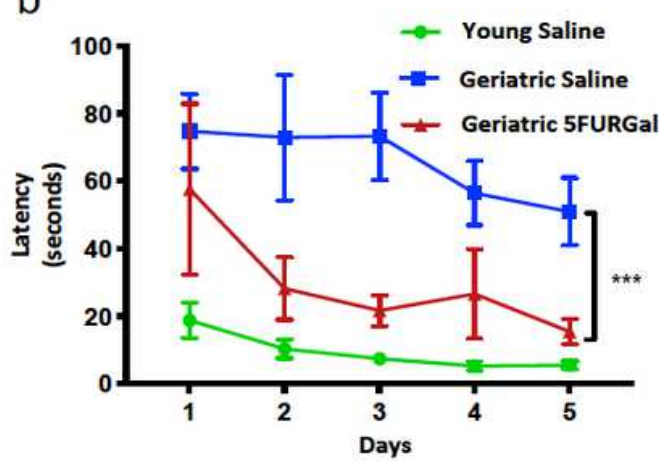

$\mathrm{e}$

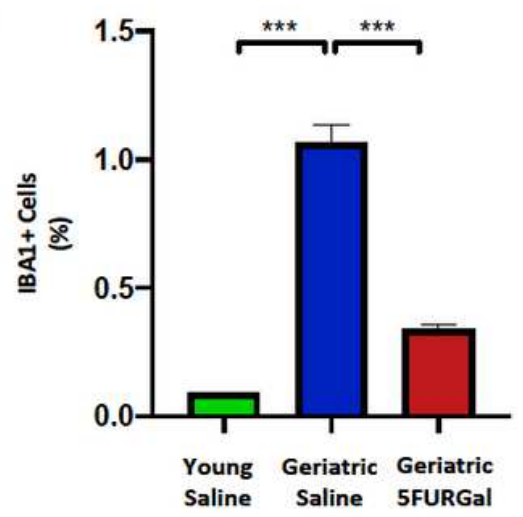

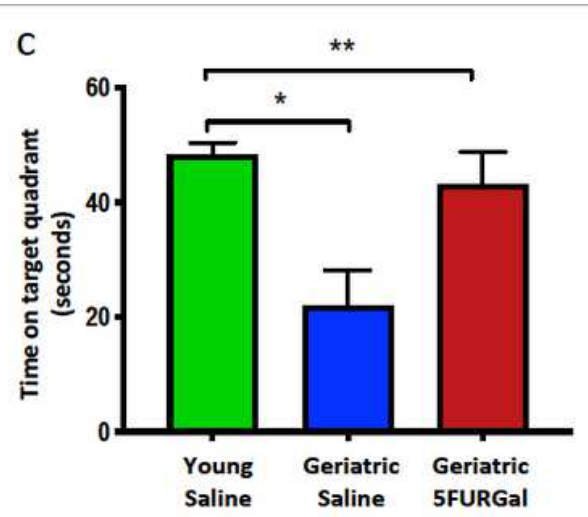

$f$

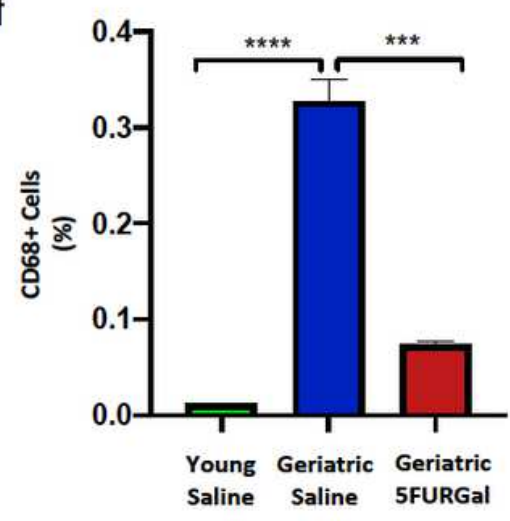

Fig 5

Figure 5 
Prodrug senolytic treatment improves cognitive functions in geriatric mice. a. Spatial Learning. Geriatric mice were subjected to $Y$ maze test before and after treatments. Graph shows fold changes of spontaneous alternation performance score $(n=6)$. b. Learning Memory. Following treatment with saline or 5FURGal and Y Maze test, young and geriatric mice were subjected to Morris Water Maze. After one week of acclimation and training, mice swimming on a pool were assessed for their trajectory to learn the position of a platform hidden on a fixed position below the surface of the water $(n=6)$. c. Memory Retrieval. Following the learning memory test shown in (b), mice were tested for the capacity to remember the original position of the platform removed from the pool $(n=6)$. $d$. Representative images of immunofluorescence (IF) of brain cross-sections showing microglia in the hippocampal regions. Brains were isolated from the mice tested for cognitive functions assessments indicated in ( $a, b$ and $c$ ), (scale bar $=2000 \mu \mathrm{m}$ ). IF was performed to stain nuclei (DAPI) and markers of microglia (CD68 and Iba-1). Quantification of IF for total microglia (e) and activated microglia (f) in the hippocampal regions of young or geriatric mice that received treatments with saline or 5FURGal and assessed for cognitive functions $(n=4)$. Data are \pm s.e.m. For statistical analysis, t-tests were used. For graph of panel $(b) 2$-Way ANOVA was used. ${ }^{*} \mathrm{P}<0.01,{ }^{* *} \mathrm{P}<0.001,{ }^{*} * \mathrm{P}<0.0001, * * * * \mathrm{P}<0.00001$.

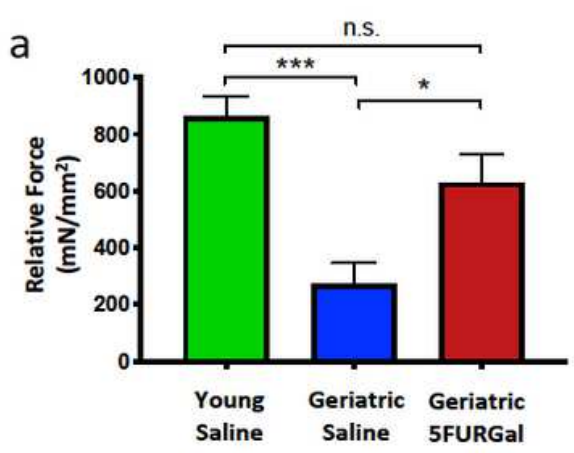

d

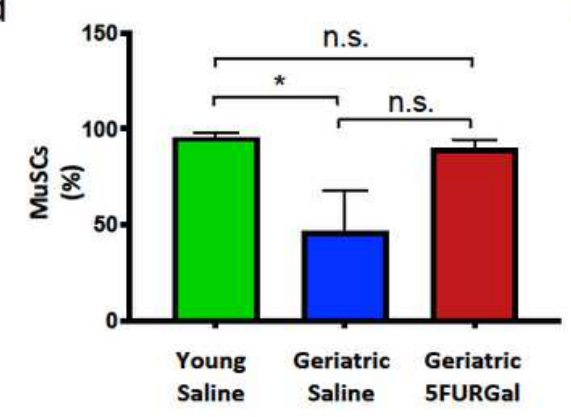

b

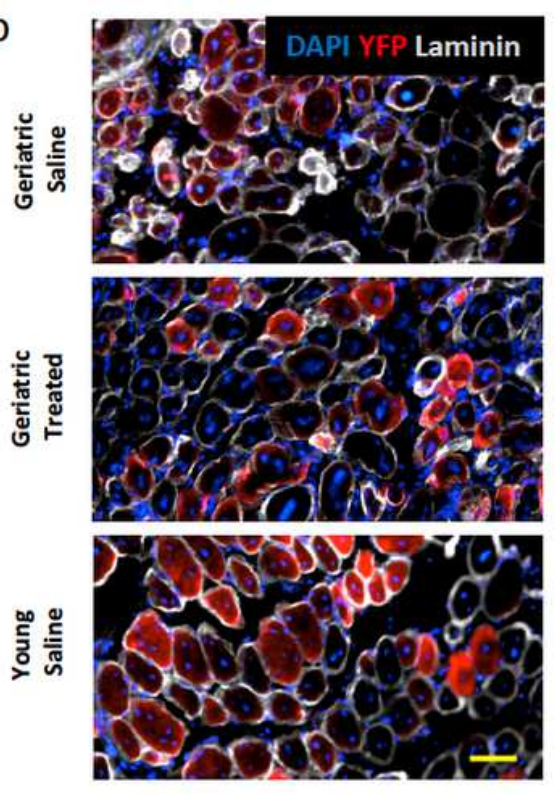

e

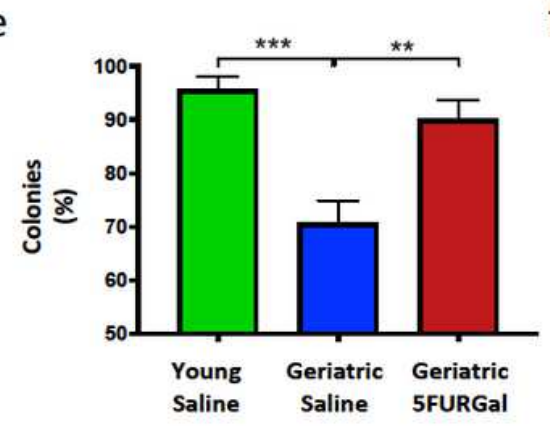

C
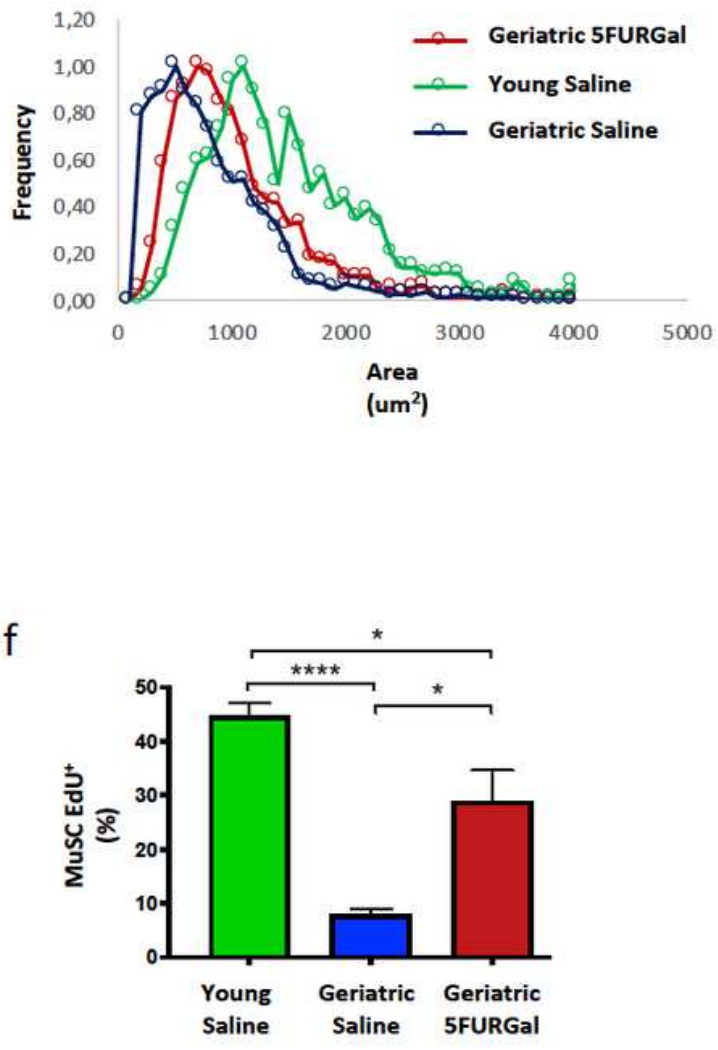

Fig 6

Figure 6 
Prodrug senolytic treatment improves tissue and stem cell muscle functions in geriatric mice. a. Tetanic force production of young and geriatric mice treated with saline or 5FURGal. Following treatments and were tested for frailty, functional and cognitive functions, mice received an injection of CTX in the TA muscles to induce MuSCs activation and muscle regeneration. After 10 days, TA muscles were dissected and the tetanic force measured ex vivo (TA muscles from mice: young treated with saline $n=9$; geriatric treated with saline $n=5$; geriatric treated with $5 F U R G a l n=6$ ). b. Representative IF cross-sectional images showing centrally nucleated regenerated YFP+ myofibers. Muscles from young or geriatric mice were harvested after taking force measurements (scale bar $=500 \mu \mathrm{m}$ ). c. Quantification of the cross-sectional area of centrally-nucleated YFP+ fibers in regenerating TA muscles that received CTS injections $(n=4)$. $d$. Quantification using fluorescent activated cell sorting (FACS) of MuSCs YFP+ isolated from injured TA muscles that received CTW injections (biological replicates $n=4$ ). e. Myoclonal assay of MuSCs YFP+ isolated from injured TA muscles that received CTW injections. Single MuSCs were isolated using FACS in a 96 well plate and quantified for their capacity to generate colonies (biological replicates $n=4$ ). f. Cell cycle assessment. MuSCs isolated from injured TA muscles that received CTW injections were cultured in the presence of EdU and quantified (biological replicates $n=4$ ). For statistical analysis, $t$-tests were used. ${ }^{*} \mathrm{P}<0.01,{ }^{* \star P} \mathrm{P}<0.001,{ }^{* \star *} \mathrm{P}<0.0001,{ }^{* * *} \mathrm{P}<0.00001$.

\section{Supplementary Files}

This is a list of supplementary files associated with this preprint. Click to download.

- NatureAgingManuscriptSenolyticprodrugingeriatricmodelsSupplementalFigures.pdf 